\title{
DALL'EPISTOLARIO DI CARLO ERMES VISCONTI, NOTE SU VICENDE DI MONUMENTI MILANESI
}

\author{
MICHELA MARISA GRISONI (*) \\ Nota presentata dal m.e. Amedeo Bellini \\ (Adunanza dell'8 maggio 2014)
}

SunTO. - Le inedite lettere dell'epistolario di Carlo Ermes Visconti (1834-1911) stimolano un riesame di alcune questioni edilizie milanesi discusse negli ultimi decenni dell'Ottocento ed in particolare quelle relative al Lazzaretto e al restauro del Castello Sforzesco. La corrispondenza del nobiluomo, tra gli altri con Luca Beltrami, Gaetano Negri, Giovanni Celoria, mostra la natura dei suoi legami con l'ambiente culturale e politico coevo, contribuendo a definirne il profilo, non solo biografico. I contenuti e le correlazioni suo tramite individuate tra i soggetti, i fatti e le circostanze, consentono quindi di proporre alcune precisazioni ma anche talune prospettive interpretative intorno al pensiero di Visconti sul restauro e sulla tutela dei monumenti nonché al suo contributo nella formazione delle civiche raccolte municipali; due aspetti che si ritengono saldamente correlati.

ABSTRACT. - The unpublished letters of Carlo Ermes Visconti (1834-1911) goad a further investigation on the architectural events discussed in Milan during the last decades of the nineteen century, especially, referring to the Lazzaretto and the restoration of the Sforza Castle. The correspondence of the marquis Visconti, with Luca Beltrami, Gaetano Negri and Giovanni Celoria, at least, shows the nature of his relationship with the cultural and political milieu he attended supporting a deeper analysis of his profile, not only the biographic one. Contents and correlations singled out throught this documents among figures, actions and circumstances allow a few remarks and some new perspectives about his opinion on restoration and preservation of the ancient buildings and, furthermore, the role he played in the settlement of civic collections and museums, being these topics different part of a same goal.

(*) Politecnico di Milano, Italy.

E-mail: michela.grisoni@polimi.it 


\section{UN DECENNIO TRA GLI AMMINISTRATORI DELLA CITTÀ (1882-1893)}

Carlo Ermes Visconti si approssima ai cinquant'anni quando, nel giugno 1882, è tra gli eletti al consiglio municipale di Milano. ${ }^{1}$ Nobiluomo, formatosi nelle scienze giuridiche presso l'ateneo pavese, è un possidente dedito alla gestione di rassicuranti rendite fondiarie e finanziarie oltre che un carismatico protagonista dei più noti salotti cittadini. ${ }^{2}$ E' al suo secondo mandato; ma se nel primo, risalente al quinquennio 1863-68, pare assumere impegni politici legati all'estrazione sociale e conseguenza del sostegno garantito agli ambienti patriottici negli anni risolutivi dell'unificazione del Regno, in questo mostrerà una più matura coscienza politica, una più grande carica di intenti. ${ }^{3}$ Consigliere nel primo biennio, il 25 aprile 1884 accetta la nomina di assessore per il riparto VI, dedicato all'istruzione. ${ }^{4}$ Tra rinnovi e rielezioni manterrà l'incarico fino al $1889 ; ;^{5}$ sebbene candidato, non verrà infatti rieletto anche se, conservando l'ufficio di soprintendente scolastico, di fatto rimarrà in commissione studi almeno fino al $1894 .{ }^{6}$

1 Entra ultimo in elenco con 3075 preferenze, Atti del Municipio di Milano, (1882/83), I, VIII.

2 Esponente di un' antica casata milanese che ha costruito saldi legami parentali con i Vimercati - Sanseverino e i Casati e coltiva solide relazioni amicali con i ViscontiVenosta e i D'Adda Salvaterra, tra i frequentatori del salotto di Clara Maffei e tra i protagonisti della Milano del Risorgimento è per questo che è stato perlopiù ricordato; cfr. Barbiera 1901, 200 e Visconti Venosta 1904, 647. Recentemente se ne è indagata l'attività nel campo del restauro e della tutela dei monumenti rivelando anche precoci azioni a favore delle 'bellezze naturali', cfr. Grisoni 2014b con bibliografia precedente; vi corre parallela l'analisi dell'operato a favore delle collezioni e dei musei cittadini, cfr., in particolare, Basso 2014.

3 Nel primo mandato (1863-1868), risalente alla Giunta di Antonio Beretta di cui supera le dimissioni, assume per breve tempo la carica di assessore supplente (nomina del 18 luglio 1867 dimissioni del successivo 7 agosto), Atti del Municipio di Milano, (1867), 388-403 e ibidem, 404.

4 Atti del Municipio di Milano, (1883-1884), I, VIII; 413.

5 Nelle due elezioni generali precedenti (11 gennaio 1885 e 8 maggio 1887) invece era stato rieletto e varie erano state anche le riconferme in Giunta, Atti del Municipio di Milano, (1884-1885), IX; XI; ibidem, (1885-1886), VIII; XI; ibidem, (1886-1887), VIII; XI; ibidem, (1887-1888), VII; XI; ibidem, (1888-1889), VIII-IX; XI; 2.

6 cfr. Guida di Milano, ad annum; i rinnovi trovano puntuali conferme in Archivio Visconti di San Vito di Somma Lombardo (d'ora innanzi AVSV), cas. 116, 
La permanenza di Visconti tra gli amministratori della città copre quindi più di un decennio. Si svolge nel succedersi delle giunte di Giulio Belinzaghi (1868-1884; 1889-1892), Gaetano Negri (1884-1889) e Giuseppe Vigoni (1892-1899); anche se si documentano corrispondenze più fitte con il secondo della terna, vicino a Visconti anche per un'affinità di interessi che accosta entrambi a quell'ambiente tecnicoscientifico milanese che gravita intorno al Museo di Storia Naturale e al suo direttore, l'abate e paleontologo Antonio Stoppani, al Regio Istituto Tecnico Superiore e a Francesco Brioschi, il celebre matematico nonché direttore della scuola e presidente del Consorzio degli istituti di istruzione superiore. ${ }^{7}$ Può dunque definirsi una presenza di un certo spessore, per durata e continuità; rivelatrice, per legami e corrispondenze, di un attaccamento agli ambienti dei conservatori milanesi, dove gli accenti progressisti, quando condivisi, assumono toni moderati e il liberalismo, pure ben presente, esprime radicate eredità illuministe.

Il suo secondo mandato, in particolare, e si tratta dell'aspetto che più interessa, si confronta con la definizione del volto 'moderno' di Milano. E' in questi anni che si edificano le aree di espansione, con il conseguente ridisegno della trama urbana e delle rendite di posizione; che si promuove il potenziamento delle infrastrutture e dei servizi e si interviene sull'immagine del centro cittadino. Il tutto si affronta attraverso la redazione di uno strumento, il piano regolatore, destinato a disciplinare la progettazione urbana. Prodotto tra 1884 e 1889 dall'ufficio tecnico municipale, il cosiddetto 'piano Beruto', notoriamente il primo predisposto per Milano, fu lo strumento consapevolmente voluto dal Sindaco e dalla Giunta che subentrarono a Belinzaghi dopo le sue roboanti dimissioni. ${ }^{8}$ E' stato sagacemente additato il rapporto di dipendenza tra scandalo e decisioni urbanistiche che pervade il clima della Milano post-unitaria in base al quale il piano regolatore sarebbe stato uno strumento di difesa dell'amministrazione pubblica: il mezzo con cui ricondurre sul piano della legalità e dell'interesse collettivo il progetto urbano; anche se i risultati si giudicano meno eclatanti delle premesse."

fasc. 1892 e fasc. 1894. Per il significato della Commissione studi in rapporto alla legge Casati (13 novembre 1859) e nel mandato di Belinzaghi, cfr. Finocchi 1978.

7 Grisoni 2012.

8 Rossari 1997 e Boriani, Rossari 1992.

9 Vercelloni 1991, 153. L'autore sottolinea la qualità dell'originaria proposta di 
Per quanto Visconti paia semplicemente assistere alla messa a punto del piano, garantendo una presenza costante alle sedute del consiglio ma trattenendosi in un riserbo pacato nelle discussioni che più direttamente lo riguardano, egli appartiene alla Giunta che ne approva la messa in atto. Accertare le sue effettive responsabilità, nel lungo percorso di gestazione, è un obbiettivo temerario per molte ragioni, non ultima l'impossibilità di accreditare responsabilità individuali alle decisioni sostenute da un gruppo politico sostanzialmente compatto; quello che si vuole tentare è di integrare le rare e pacate esternazioni istituzionali del marchese con le inedite corrispondenze epistolari che ne svelano le relazioni e le confidenze.

La redazione del primo piano regolatore cittadino inoltre interseca il tema del restauro dei monumenti ed egli, in particolare, si confronta con le responsabilità pubbliche nella gestione dei luoghi storici della città e dei suoi 'avanzi' monumentali.

Per Visconti la tutela dei monumenti è un'attività alimentata da un forte senso civico; l'espressione coerente e diffusa dell'accostarsi degli uomini colti al patrimonio storico e artistico come simbolo di identità nazionale e strumento di elevazione sociale. Persiste, inoltre, la convinzione della funzione didattica delle opere del passato per quelle presenti, retaggio della cultura positivista in cui egli è cresciuto. ${ }^{10}$ Senza esprimersi in elaborazioni teoriche (che mancano nei suoi scritti e sostanzialmente non appartengono alla natura del suo profilo) questo pensiero affiora riesaminando, attraverso le sue carte, alcune note vicende edilizie cittadine.

\section{IN RISPOSTA AI NUOVI QUARTIERI AL LAZZARETTO (1878-1881)}

All'arrivo di Visconti in Consiglio comunale, nel giugno del 1882,

Cesare Beruto definendolo un piano colto e rilevando nella bocciatura del consiglio l'incapacità di capirne se non di accettarne l'impronta ideologica sottoponendolo, attraverso la revisione del consigliere Giovanni Battista Pirelli, ad uno 'svilente' depauperamento dei contenuti di maggior pregio.

10 Prima di accedere agli studi universitari in giurisprudenza presso l'Ateneo pavese, Carlo è istruito da precettori privati sotto la vigile tutela della madre, Leopolda Isimbardi, e di Paolo Taverna. Si ricorda inoltre che egli è il nipote del letterato Ermes Visconti, fratello di suo padre Giuseppe. Alcune lettere dello zio al nipote in AVSV, cas. CC. 
la 'questione' del Lazzaretto è già stata dibattuta ${ }^{11} \mathrm{e}$ oramai irrimediabilmente orientata verso la soluzione tendente a soddisfare le parti coinvolte mosse dalla redditività dell'operazione immobiliare che è alla base. E' stato osservato che l'amministrazione comunale sembrò assistere alla trattativa con piatta rassegnazione; senza curarsi, o avvalersi, del titolo di edificio monumentale assegnato al Lazzaretto nel $1875 .{ }^{12}$

Nelle dinamiche urbane questa architettura subì infatti gli effetti dell'inclusione, entro i confini della città, del comune dei Corpi santi in cui si trovava fatalmente a ricadere. La rivalutazione dei valori di rendita fondiaria delle aree prima appartenenti alla cintura esterna della città incrementò la loro appetibilità in un mercato immobiliare i cui operatori reagirono con pron tezza e determinazione, ansiosi di recuperare questo suolo per sostituire al vasto quadrilatero quattrocentesco che ne disegnava il perimetro (e in parte già compromesso dal tracciato ferroviario), una volumetria più densa, da elevarsi su una trama viaria più fitta. Quella del Lazzaretto «lasciato al suo destino tra deboli rimostranze, non appena una società finanziaria ne propone la distruzione e la lottizzazione» ${ }^{13}$ è considerata pertanto dalla storiografia una vicenda esemplare delle contraddizioni presenti nella cultura urbana di secondo Ottocento: indicativa della difficoltà di conciliare progresso e tradizioni, principi economici e valori culturali.

Esaminando la vicenda al 1882 si vedrà quindi che per il Consiglio in cui siede Visconti si tratta oramai di indirizzare e convalidare le proposte dei tecnici esterni chiamati a redigere il progetto degli edifici che l'amministrazione si è riservata come traguardo di pubblica utilità nell'ambito di un'operazione finanziaria tra privati di manifesto carattere speculativo; si tratta della realizzazione delle scuole elementari di via Tadino, tuttora esistenti. ${ }^{14}$ Come assessore alla pubblica istruzione Visconti interverrà nella questione a questo proposito: per esprimersi sulla qualità del noto disegno di Angelo Savoldi, contribuendo peraltro a difenderlo alle critiche del consigliere De Cristoforis. ${ }^{15}$

Questo ruolo, dal carattere apparentemente incidentale, muta quando si riesamini la vicenda da un altro punto di vista, anche cronologico.

11 Atti del Municipio di Milano, (1882-1883), 125: Seduta del 2 gennaio 1882.

12 Treccani 1992, 250.

13 Boriani 1992, 393.

14 Giustina 1991, 198.

15 Atti del Municipio di Milano, (1885-1886), I, 358-361: Seduta del 25 febbraio 1886. 
Membro della Commissione conservatrice dall'aprile del $1878,{ }^{16}$ Visconti infatti è tra coloro che avevano respinto un progetto, presentato dal proprietario, tendente a modificare il fronte dell'edificio verso il corso Loreto. Tra coloro cioè che, edotti dalla relazione dell'architetto Angelo Colla, altro (ed influente) dei membri della commissione, avevano deciso di accogliere «per ogni parte» il parere negativo già espresso dal Municipio e di appoggiarlo non solo con «una deliberazione identica» ma insistendo «a tutto potere sulla necessità di non lasciar più oltre deturpare un Monumento storico di tanta importanza com'è il Lazzaretto». ${ }^{17}$ Schierati su un compatto fronte comune, amministrazione municipale e commissione conservatrice, nel dicembre del 1878 si erano infatti irrigiditi su una posizione di stretta conservazione dell'intero edificio. ${ }^{18}$

Le circostanze erano mutate quando, nel 1880, il Consiglio degli istituti ospedalieri (proprietario dell'immobile) non si faceva più promotore di un progetto di trasformazione ma si era risolto a cedere la proprietà ad altri e di questo informava la commissione la quale non aveva mezzi per opporsi al diritto di un privato di alienare un proprio bene. Commissione e Comune si erano dunque trovati a fronteggiare le pressioni di un grosso operatore finanziario (la Società del Credito Italiano) il cui progetto avrebbe peraltro contribuito a riqualificare un'area urbana degradata. Tale infatti appariva il Lazzaretto nel 1880; ed è aspetto non irrilevante per la successiva risoluzione della vicenda. Affacciata sul corso Loreto ma posta a ridosso dei caselli di Porta Venezia (e quindi al viale di accesso orientale al centro città), vicina ai giardini pubblici (e quindi alla sede dell'imminente Esposizione Nazionale), l'architettura anticamente progettata per ricoverare gli appestati e ora occupata da botteghe artigiane e modesti alloggi in affit-

16 Atti del Municipio di Milano, (1877-1878), I, 160: Seduta del 19 aprile 1878. Visconti è eletto con voti 27 su 45. Per l'elenco delle sedute cui ha partecipato e degli incarichi conferiti si rimanda a Grisoni 2008.

17 Atti I 1880, 58: Seduta del 21 dicembre 1878, punto 14 cui si riferiscono tutti i virgolettati del paragrafo.

18 Approvate le conclusioni proposte dall'architetto Colla nella sua relazione, i commissari si erano infatti associati «al Municipio nel concetto di impedire che per l'avvenire si permettano modificazioni e costruzioni, le quali deturpino o alterino il carattere storico di quel vasto edificio», ivi. Compongono la commissione Isaia Graziadio Ascoli, Michele Caffi, Carlo Casati, Pompeo Castelfranco, Angelo Colla, Felice De Maurizio, Tullo Massarani, Giuseppe Mongeri e Carlo Ermes Visconti; al voto sono tutti presenti. 
to, appariva fuori luogo: sacrificabile a fronte delle legittime aspirazione economiche di entrambi i contraenti e delle positive ricadute di decoro pubblico perseguibili con l'abbattimento.

$E^{\prime}$ in questo scenario che operò la sotto-commissione, nominata in seno alla Commissione Conservatrice, incaricata di riesaminare il caso e di riferirne «un motivato parere». ${ }^{19}$ Composta da Giuseppe Colombo, Carlo Casati, Angelo Colla e dallo stesso Visconti essa si espresse nella seduta del 14 dicembre 1880 (quella in cui i commissari accettarono la demolizione dell'edificio con quella prosaica rassegnazione sottolineata dalla critica). ${ }^{20}$ Nella decisione, posta al di fuori della conservazione dell'immobile nel suo complesso, è già stato colto l'affidarsi ad un criterio di selezione delle parti da conservare fondato sulla ripetitività degli elementi architettonici (il sistema edilizio porticato verso l'interno del recinto) e basato sull'accertamento di qualità artistiche (i rilievi in cotto delle aperture e le decorazioni pittoriche eventualmente rinvenute). L'architettura del Lazzaretto fu considerata tale cioè per cui si potevano conservare alcuni elementi tipici (qualche arcata esemplificativa), magari riuscendo ad includerli nella costruzione dei nuovi edifici (proposta di Tullo Massarani) e (qualora si fossero ritrovate) recuperando le pitture votive menzionate nei documenti d'archivio (avvertimento di Carlo Casati). Si conservò invece la chiesa centrale la cui importanza fu sottolineata da Massarani, condivisa da Giuseppe Colombo e sottoscritta dallo stesso Visconti. Motivazioni di carattere spirituale concorsero infatti a garantirne la sopravvivenza che, sul piano pratico, comportava minori vincoli alla nuova edificazione. ${ }^{21}$

Si è ricordato che la demolizione del Lazzaretto dimostrerebbe la debolezza dei coevi organismi di tutela, la carenza di un'efficace normativa che fosse loro di supporto nel fronteggiare le pressioni esercitate sugli operatori dalle forze locali. Rivelerebbe, soprattutto, la difficile

19 Atti II 1881, 17-18: Seduta del 14 dicembre 1880, punto 8.

20 Nell'occasione Visconti è incaricato, con Casati e Colombo, di prendere in esame la proposta vendita del castello di Trezzo, AVSV, cas. 115: Il prefetto di Milano, Achille Basile, a Carlo Ermes Visconti. Lettera del 7 dicembre 1880 su carta intestata Prefettura della Provincia di Milano.

21 Aggiunta all'edificio quattrocentesco, parzialmente riconfigurata come monumento dei milanesi scampati alla peste, poi dedicata alla memoria di San Carlo, è un'architettura di dimensioni più contenute, Atti II 1881, 17-18: Seduta del 14 dicembre 1880 , punto 8 . 
conciliazione, tra diritto pubblico e privato; dunque l'urgenza di intervenire per aumentare l'autorità degli organismi di tutela, allora ancora a carattere consultivo, e forse per alcuni l'intuizione dell'importanza di regolare la prevalenza degli interessi economici a fronte di valori di altra natura. E' di un certo interesse capire in quale misura ne fossero consapevoli i protagonisti e, in particolare, quanto questa vicenda abbia influito sul percorso di Visconti e sulla decisione della sua candidatura politica.

Occorre quindi ricordare che durante lo svolgersi dei fatti la composizione della commissione muta e notare che alla seconda occasione di discussione parteciparono alcune delle personalità già presenti nel 1878, ma non tutte. Tra gli allontanamenti volontari spicca quello di Giuseppe Mongeri, 'maturo' storico e critico dell'arte, manifestamente espressosi con disappunto anche su questi argomenti, le cui dimissioni vengono presentate proprio nel giorno della discussione. ${ }^{22} \mathrm{Nel}$ marzo successivo giungeranno anche quelle di Visconti. Si giustificano in rapporto all'incompatibilità dei suoi numerosi incarichi ${ }^{23}$ ma le ripetute presentazioni, i relativi rinvii e le dense minute conservatesi tra le sue carte, confessano un disagio più profondo. E' sostenuto da sostanziali divergenze di vedute; da rileggere, si crede, richiamando i pregressi conflitti che avevano opposto la Consulta del Museo Patrio alla nascente Commissione conservatrice milanese (1876-77) e, soprattutto, la contestata nomina dell'ispettore onorario (1875) e quindi questioni quali l'autorità e titolarità dei rispettivi membri, la gestione del Museo Patrio, il progetto, già latente in quegli anni, dell'aggregazione di quest'ultimo a quello artistico (1879-1881). ${ }^{24}$ Seguiranno quelle di Massarani (che quella confessione aveva ricevuto); poi di Angelo Colla e Isaia Graziadio Ascoli. La rosa dei rimanenti

22 Atti II 1881, 11: Seduta del 14 dicembre 1880, punto 1.

23 Per giustificare le proprie dimissioni Visconti, da poco subentrato al defunto Paolo Belgiojoso tra gli amministratori della Veneranda Fabbrica del Duomo, avanza il motivo dell'incompatibilità dei due ruoli, cfr. AVSV, cas. 115.

${ }^{24}$ Per una ricognizione della carte relative al progetto di aggregazione, significativamente giunto, al 5 novembre 1881, alla proposta di radunare i due musei, archeologico ed artistico, al Castello ma anche per le pressioni esercitate da Pompeo Castelfranco, a più riprese, sia presso le sedi ministeriali che sullo stesso Visconti e tendenti alla creazione di un museo paletnologico di cui si candida direttore, cfr. La Guardia 1989, 10-14 ma anche La Guardia 1983, n. 75.01/1-3. L'epistolario privato spinge infatti ad approfondire le intese tra i soggetti coinvolti fin dal 1877. Si veda per questo anche Basso 2014, 171. 
mostra le figure emergenti. ${ }^{25}$ I fatti successivi dimostreranno che non si tratta di insanabili fratture ma dell'avvio di un radicale ripensamento trasversale tanto alla teoria del restauro dei monumenti che alla gestione della loro tutela.

Mentre maturavano questi nuovi indirizzi, il destino del Lazzaretto si era però dovuto decidere, sotto la pressione degli interessi in gioco, nel rispetto degli strumenti di tutela disponibili. La commissione conservatrice si era quindi risolta a formulare collegialmente il proprio parere consultivo con un'espressione favorevole all'alienazione, ponendo però alcune condizioni. La 'conservazione' del Lazzaretto fu cioè riposta nelle pieghe delle clausole contrattuali che suggerivano cautela nelle demolizioni ${ }^{26}$ e che, prima di tutto, chiedevano il rilievo dell'edificio $^{27}$ come noto, affidato a Luca Beltrami. ${ }^{28}$

25 Sulla cosiddetta 'ondata dimissionaria' cfr. Savare' 1994, 244-246. Si nota che ai dimissionari Mongeri e Visconti subentreranno Giuseppe Bertini e Camillo Boito, Atti del Municipio di Milano, (1880-1881), I, 268: Seduta del 13 aprile 1881.

26 Del Lazzaretto di Milano oggi, lo si ricorda, rimangono in loco la chiesa centrale dedicata a S. Carlo (un poco trasformata) e un tratto del muro esterno nord-est con relativo porticato interno lungo la via S. Gregorio; persiste inoltre il toponimo ad identificare la strada che con le attuali vie S. Gregorio, corso Buenos Aires e viale Vittorio Veneto consente di riconoscerne il recinto. Frammenti degli apparati decorativi giunsero invece al Museo Patrio di Archeologia, come si deduce dall'inventario dell'archivio del museo (La Guardia 1989), e precisamente due lapidi, depositate nel luglio del 1882 (ivi, n. 558), un busto raffigurante la Carità acquistato nel corso delle demolizioni e ceduto dagli acquirenti al Museo nell'ottobre del 1886 (ivi, nn. 633 e 635), una lapide in cotto rinvenuta durante le demolizioni nel 1890 e donata al museo nel 1894 dall'antiquario Carlo Clerici (ivi, n. 1109).

27 «La Commissione risponderà al Consiglio Ospitaliero, che essa, non opponendosi alla vendita del Lazzaretto, lo prega di voler far eseguire e trasmettere alla Commissione stessa l'Icnografia generale, la Sciografia e l'ortografia del Lazzaretto, come si trova attualmente; e inviterà l'opera pia a riservarsi rimpetto all'acquirente il diritto di esportare una lapide esistente sulla parte interna della porta del Lazzaretto, verso la circonvallazione, e altri cimelj che si potessero rinvenire nella demolizione, allo scopo di poi trasmettere tutti codesti cimelj, come anche qualche saggio delle decorazioni del portico, al Museo Patrio di Archeologia. La Commissione raccomanda infine, che nelle opere di demolizione si proceda con la massima cautela, perché consta che in alcune camere del Lazzaretto esistono pitture del tempo», cfr. Atti II 1881, 18: Seduta del 14 dicembre 1880, punto 8. Nella seduta del 19 aprile 1881, la commissione, ormai mutata nella sua composizione, visionerà i disegni presentati dal segretario Fossati, cfr. Atti II 1881, 27-38: Seduta del 19 aprile 1881, punto 6.

28 Oltre ai rilievi per il Lazzaretto, conclusi entro la fine del 1881, Beltrami 


\section{DA CONSIGLIERE AD ASSESSORE DEL RIPARTO VI - ISTRUZIONE (1882-1884)}

Nel 1881 Beltrami è un giovane ma già conosciuto architetto da poco rientrato a Milano da un soggiorno parigino professionalmente formativo che si presenta alla pratica professionale da vincitore di un celebre concorso cittadino e si appresta ad assolvere compiti didattici presso l'Accademia di Brera dalle quale formalmente giunge l'incarico del rilievo del Lazzaretto. ${ }^{29}$ All'anagrafe lo separano vent'anni dal più anziano marchese Visconti; ${ }^{30}$ ma negli anni Ottanta dell'Ottocento tale distanza si contrae e a Milano, ove entrambi risiedono, gli ambienti frequentati e le relazioni comuni offrono occasioni di incontro, rafforzate dalla corrispondenza epistolare. I contatti documentati richiamano titoli, luoghi e temi che riconducono distintamente al mandato del «caro Visconti» cui Beltrami si rivolge non solo come ad un collega ma perlopiù come ad un confidente che raggiunge in ufficio per «bagolare un poco» dei suoi affari $;{ }^{31}$ si possono cioè fondatamente ricondurre all'ambito di un comune impegno politico le relazioni tra Visconti e Beltrami, ugualmente presente in Consiglio Comunale, ma dal gennaio del 1885, e soprattutto ugualmente giuntovi con il sostegno dell'Associazione costituzionale..$^{33}$

Gruppo politico cui Visconti si accosta fin dal 1870, l'Associazione costituzionale era favorevole ad un modello liberale monarchico di gestione dello Stato. A Milano contò sull'appoggio de 'La Perseveranza'; ma per le elezioni dell'82, quelle che riportarono Visconti in Consiglio comunale, il consenso si era decisamente allargato e tutti, dal 'Corriere

compose il testo descrittivo dell'edificio da includere nella guida di Milano pubblicata per l'Esposizione del 1881, cfr. Beltrami 1881. I disegni, con un corredo testuale più ricco, sono riprodotti in Beltrami 1882, 425-426.

29 Per un profilo sulla figura di Luca Beltrami, tra i molti contributi dello stesso autore, si indica il più recente Bellini 2014 ove si deduce anche la bibliografia precedente. Per i compiti didattici si veda anche Ricci 1997, 146.

30 Visconti nasce il 25 novembre 1834, Beltrami il 13 novembre 1854.

31 in Appendice, doc. 3.

32 Atti del Municipio di Milano, (1884-1885), I, XIII. E’ eletto il giorno 11 gennaio 1885 .

33 Beltrami è incluso, insieme ad altri - tra i quali lo stesso Visconti - nella celebre Lista Unica proposta dal Circolo della Costituzionale, La Perseveranza, 4 gennaio 1885 . 
della Sera' fino a 'Il Pungolo', concordarono nel sottoscrivere la lista presentata da Francesco Brioschi. Visconti vi era stato incluso: segnalato agli elettori come un cittadino stimato, affidabile e, precisava l'illustre professore, già molto coinvolto nelle «questioni edilizie della città». ${ }^{34}$ I moderati de 'La Perseveranza' vi riconoscevano l'interprete degli interessi dei possidenti. ${ }^{35}$ Cauto progressista e conservatore avveduto, Visconti quindi aveva goduto di un'ampia base di appoggio.

Passati due anni dal suo ingresso in Consiglio e, soprattutto, dopo tre roventi giorni di discussione sui progetti per i nuovi quartieri alla piazza d'Armi, il 18 aprile 1884, Sindaco e Giunta come noto si dimisero. Il discorso di insediamento dell'assessore anziano, Gaetano Negri, oltre a mostrare gli elementi di continuità con i predecessori, chiarendo lo scenario politico-finanziario di sfondo alla crisi, aveva fissato l'ordine delle priorità stabilito dalla sua Giunta. ${ }^{36}$ Si deriva da quel discorso che si trattava di un trasferimento di mansioni, un passaggio di mandato più che un avvicendamento, in cui Negri subentrava a Belinzaghi e Visconti, in particolare, a Negri nel ruolo di assessore all'istruzione e soprintendente scolastico.

34 Per presentare agli elettori Visconti, Francesco Brioschi disse: «Il marchese Visconti Ermes è una delle individualità più spiccate della classe alla quale appartiene. La sua dottrina artistica ed archeologica lo designava già ad amministratore della fabbrica del Duomo, a socio onorario residente dell'Accademia di Belle Arti, a commissario pel nostro Museo artistico Municipale. In questi uffici, senza parlare di altri che non hanno rapporto colla questione edilizia, il marchese Visconti Ermes si dimostrò sempre collaboratore attivo ed intelligente. Proponendovi la sua candidatura, abbiamo voluto onorare in lui il cittadino che modestamente pone a servigio del proprio paese i suoi studi ed il suo tempo, senz'altro compenso che la soddisfazione di aver adempiuto ad un dovere, e nello stesso tempo esplicare sempre più dei criteri adottati inviando al Consiglio un uomo che ha già tanta parte in questioni edilizie della nostra città». Oltre al nome di Visconti, la lista della Costituzionale includeva: Tommaso Bertarelli, Gerolamo Chizzolini, Francesco Lovati, Giovanni Battista Pirelli, Edoardo Porro, Archimede Sacchi, Paolo Manusardi, La Perseveranza, 7 giugno 1882.

35 «A tutto ciò che nella Relazione [Brioschi] è detto della candidatura dell'Ermes Visconti, si deve aggiungere che egli è anche possidente nella nostra città, e come tale rappresenterà anche questa parte capitale dei contribuenti che sono i possessori di case. C'è una soverchia e dannosa tendenza a dimenticare che le rappresentanze amministrative sono essenzialmente rappresentanze d'interessi; è bene correggerla», La Perseveranza, 10 giugno 1882.

36 Atti del Municipio di Milano, (1883-1884), 417-421: Seduta del 12 maggio 1884. 
Legati da un rapporto di stima reciproca, risalente di qualche decennio e riconducibile al circolo dei naturalisti e degli scienziati milanesi, Visconti e Negri condividono il progetto di una nuova sede per il museo di storia naturale che è una delle priorità indicate; un progetto che Visconti aveva visto maturare accostandosi a quegli ambienti fin dagli anni sessanta. ${ }^{37}$ Nei programmi della Giunta guidata da Negri, mentre si frenava lo sviluppo dell'area nord-ovest anteponendovi come pregiudiziale la redazione del piano regolatore, si affrontava direttamente la questione. Non si trattava solo di un progetto edilizio (peraltro in quel momento ancora da definirsi attraverso un lungo percorso di affinamento) ma più estesamente culturale. Si è ipotizzato che mentre si ordinavano razionalmente le collezioni naturalistiche municipali, concentrandole nel settore urbano gravitante intorno ai giardini pubblici dove si trovavano gli istituiti di istruzione superiore destinati a servirsene, si favoriva la discussione sulla dislocazione dell'intero patrimonio collezionistico cittadino $;^{38}$ di quello artistico, storico e archeologico, in particolare, allora diviso tra il Salone (dove si trovavano il Museo Artistico e quello, nascente del Risorgimento) e i locali di Brera (dove era stato sistemato il Museo Patrio di Archeologia). ${ }^{39}$ Questa discussione e la ricognizione delle risorse edilizie di proprietà pubblica più adatte allo scopo sono argomenti sui quali Visconti, giunto in Consiglio comunale, si esprimerà con interventi rari ma mirati, a conferma delle parole di Francesco Brioschi.

\section{Per molti AnNi a SERVIZio Delle Civiche RaCcolte (1863-1900)}

Il Museo Artistico Municipale era stato inaugurato nel giugno 1878. Giuseppe Mongeri, puntuale cronista dei fatti culturali cittadini, non aveva risparmiato alcune critiche osservando che le sette sale del piano superiore del Salone ai Giardini Pubblici, ove erano stati disposti gli oggetti, non avevano consentito quella disposizione richiesta «da una rigorosa razionalità artistica» anche se, avvertiva, la natura del pro-

37 Grisoni 2012.

38 Grisoni 2014a.

39 Sull'istituzione del Museo del Risorgimento si veda Zatti 2012 che ne mostra la genesi come prodotto dell'Esposizione Nazionale Italiana del 1884; si osservi, inoltre, la presenza di Visconti tra i componenti della commissione milanese. 
blema non risiedeva solo nella sede - cui si era giunti consapevoli della provvisorietà della scelta - ma anche nella genesi delle collezioni pubbliche. Scriveva infatti l'affermato e noto storico dell'arte:

Quando una collezione com'è questa, altro non sia che il portato di quattro o cinque eredità, domandare un ordine logico, sistematico preciso è richiedere quello che solo il caso potrebbe dare per miracolo. Qui, l'ha proprio negato; ma la Commissione ordinatrice ha fatto del suo meglio. ${ }^{40}$

«Più che un museo fatto, un museo da creare» avrebbe ammesso poco dopo Carlo Ermes Visconti proprio dall'interno di quella citata Commissione ordinatrice. ${ }^{41}$ Egli aveva lavorato personalmente, nell'inverno e nella primavera del 1878, all'ordinamento dei materiali completando il catalogo di «alcune classi» e portando «a discreto punto», o almeno iniziando, quelli delle rimanenti ma era cosciente di offrire «embrioni di collezioni» (salvo le ricche collezioni numismatiche, il medagliere e preziose opere di grandi maestri che già potevano formare una significativa pinacoteca dello svolgersi dell'arte antica). Mostrava tuttavia un certo compiacimento (la cui enfasi è certamente strumentale alla sede e alle veste in cui si trovava ad esprimerlo) nel vedere finalmente radunati, dalle sedi più disparate, quel dono e quei legati che sono notoriamente l'origine delle civiche raccolte milanesi (la Raccolta Marchesi, i legati di Antonio Guasconi e di G.G. Bolognini Attendolo, quelli di Giuseppe Sormani e di Carlo Taverna e, infine, di Malachia De Cristoforis) e quanto nel frattempo vi si era aggiunto. ${ }^{42}$ Mentre si rendeva il dovuto onore ai donatori, si incitavano infatti altri privati ad emularli; con una precisazione sobillatrice di Visconti in merito all'importanza delle collezioni pubbliche a fronte di quelle private (ritenute di difficile sopravvivenza perché più soggette a dispersione per effetto dei nuovi quadri normativi). Si rivela così la sua attenzione per una forma di tutela del patrimonio storico ed artistico che, riconosciutone l'interesse collettivo, invoca responsabilità pubbliche. Vi si affianca un'esortazione al senso civico dei cittadini, di cui egli darà prova in prima persona con doni e legati..$^{43}$

40 Mongeri 1878.

41 Visconti 1880.

42 Mongeri 1878.

$43 \mathrm{Al}$ Museo del Risorgimento, ad esempio, dona un ritratto dello zio Ermes Visconti e un autografo di Garibaldi, AVSV, cas. 115, fasc. 1886. 
Illuminato da questi principi, Visconti si accollò un impegno di raccolta e catalogazione che lo travolse; documentato come tale dai contemporanei ${ }^{44}$ amplificato oltre misura dalla satira, confermato dalle sue carte personali le quali rivelano l'abilità di intercettare le collezioni dei privati arginando smembramenti ed esportazioni, ${ }^{45}$ di dialogare con i mercanti ${ }^{46}$

44 In «quegli anni che andarono dal 1877 al 1880 [...] il marchese Visconti ed il museo Artistico si identificavano: egli era tutto: esercitava le funzioni di direttore e di segretario, di restauratore e di scrivano», Novati 1912.

45 Visconti è il 'tramite' che presenta il legato Ponti al Comune di Milano, cui era stato destinato, AVSV, cas. 116, fasc. 1896 ma anche cas. CC, fasc. 2: Gustavo Frizzoni a Carlo Ermes Visconti, lettera del 7 settembre 1895. Nel 1899 coopera con Giuseppe Vigoni per assicurare al Comune il lascito della marchesa Stampa-Soncino, cfr. Consulta, 1284 e ss.. Più articolato il ruolo in rapporto alla collezione Passalaqua, Giunta al Comune di Milano a più riprese anche per interessamento di Visconti. Per questo si veda ora Amadini 2013, 40-48. Si aggiunge che alla morte dell'amico Passalaqua, Lodovico Pogliaghi informa Visconti che l'erede, Alessandra Negrotto, è intenzionata a privarsi di poche cose. Scrive infatti: «Ho sentito moltissimo la perdita di Passalaqua. Ella sa come gli fossi amico e conserverò sempre con riconoscenza la sua memoria. Sono contento che il Suo nome sarà bene ricordato dai pochi oggetti che ha lasciato al Museo. Ella ricorderà certamente l'arazzo fiammingo e la cassetta scolpita e il bassorilievo di P. da Vinci state esposte e fotografate nell' 81 - ma non so se conosce un gran mobile costrutto con legni vari da un Quintilio Passalaqua del 1600 - e con mille accessori interessanti - fra gli altri alcuni diaspri dipinti dal Morazzone. L'erede marchesina Alessandra Negrotto - ha intenzione di conservare intatta la villa di Moltrasio - privandosi solo dei quadri di Luino - di qualche oggetto troppo speciale da museo e, crede - della libreria», in AVSV, cas. CC, fasc. autografi. La lettera, non datata, si può trattenere tra la data di morte del conte (5 gennaio 1890) e l'accettazione del lascito, comprensivo degli oggetti indicati, da parte del comune (24 febbraio 1890).

46 Nel dicembre del 1888 Visconti partecipa alla vendita pubblica della collezione Baslini gestita dalla Casa d'Asta Sambon acquistando, per il Museo Patrio, un'ara mitriaca e due iscrizioni, cfr. La Guardia 1989, n. 193/1-4: Lettera di Carlo Ermes Visconti a Giulio Carotti del 10 dicembre 1888. Pochi mesi dopo chiede di essere autorizzato a partecipare alla vendita all'asta della collezione De Amici per assicurarsi l'acquisto di un oggetto già nella collezione Passalaqua (un bassorilievo in marmo di scuola lombarda del secolo XVI rappresentante la Madonna col Bambino e due angeli con strumenti musicali), ivi, n. 208. E' frequentemente interpellato da librai, antiquari e generici venditori. Il 10 settembre 1896, ad esempio, il librario Ulrico Hoepli, informandolo di avere acquistato un'importante biblioteca in Roma, la propone a Visconti per il Museo del Risorgimento, in AVSV, cas. CC, fasc. 2. I contatti con il mercato riguardano ovviamente anche affari privati; trattative per l'acquisto dello stemma Visconti già nel castello di Pandino, e proposto dal venditore per quello di Somma, in AVSV, cas. CC, fasc. 1872: Giovanni Ballerini, antiquario a Carlo Ermes Visconti, lettera da Lodi del 25 marzo 1872. 
e i collezionisti, ${ }^{47}$ di vigilare sul patrimonio ecclesiastico indirizzando gli oggetti verso 'asili' sicuri, ${ }^{48}$ favorendo dunque forme di pubblica raccolta ma anche di pubblica fruizione. Per Visconti la funzione didattica è infatti inscindibilmente legata al ruolo di un moderno museo; un aspetto che si riconosce non solo in rapporto alle Accademie d'arte ma anche ai luoghi di formazione degli artigiani e di produzione delle arti minori. Allenatosi nel silente lavoro, svolto nei primi anni sessanta, per Giorgio Jan prima e per Emilio Cornalia poi, nel riallestimento del Museo Civico in Palazzo Dugnani, ${ }^{49}$ interpellato per valutare i pezzi della raccolta Marchesi, ${ }^{50}$ coinvolto nella discussa acquisizione della collezione archeologica Giani-Uboldi, ${ }^{51}$ delegato al catalogo del medagliere del

47 Emblematica la proposta di acquisto, per conto del Museo Artistico, avanzata a Laura D'Adda Salvaterra e relativa ad un certo 'letto', cfr. Biblioteca Comunale di Grosio, Archivio Visconti Venosta, busta 96, fasc. 3: Carlo Ermes Visconti a Laura d'Adda Salvaterra, lettera del 29 settembre 1883 e soprattutto la successiva in cui, a rinforzare la proposta, riferisce all'amica gli entusiasti parere di Giuseppe Bertini e Luigi Cavenaghi, ivi, lettera del 18 ottobre 1893.

$48 \mathrm{Su}$ indicazione di Emilio Seletti interpella per questo la fabbriceria della chiesa di S. Babila (La Guardia 1989, n. 1525). Prove della sua sollecita vigilanza anche in AVSV, cas. CC, fasc. 1872: Antonio Caimi, segretario dell'Accademia di Brera, a Carlo Ermes Visconti, lettera da Milano del 5 dicembre 1872, in cui lo ringrazia di avere avvisato il presidente, Carlo Belgiojoso «circa la cessione che si va trattando della tavola di scuola leonardesca di spettanza della parrocchiale di Mezzana». Si tratta, verosimilmente, del polittico raffigurante La Vergine Assunta, San Giovanni Battista e Santo Stefano attribuiti a Marco d'Oggiono e ora al Museo diocesano di Milano, inv. MD 2001089.001.

49 Jan 1863.

50 Visconti è incluso «come [persona] cognita e studiosa delle arti belle» nella «nuova» commissione (composta da Giuseppe Balzaretti, Giovanni Strazza e Giuseppe Mantegazza) cui la Giunta Municipale, pressata dal donante, Salvatore Fogliani, bisognoso di liberare lo studio dello scultore per il successivo 15 settembre, affida il compito di provvedere all'urgenza del trasporto e alla dislocazione dei pezzi non destinati a far parte della collezione artistica, in AVSV, cas. 115, fasc. 1864: Lettera del Sindaco di Milano, Antonio Beretta, in data 9 luglio 1864. Al documento si accompagnano la distinta, su carta intestata della Consulta del Museo Patrio, firmata Antonio Caimi e Giuseppe Bertini in data 21 settembre 1864, degli oggetti scelti per il Museo Patrio e un Inventario degli oggetti artistici costituenti la Raccolta Marchesi, suddiviso per Categorie, recante le misure, annotato in diversa grafia e non datato; i due documenti integrano significativamente quelli indicati in Fratelli 2012.

51 AVSV, cas. 115. La corrispondenza risale al 1867; sono allegate due lettere risalenti al 1840 . 
conte Taverna ${ }^{52}$ per il cui tramite non è infondato credere si siano configurati i primi compiti istituzionali nel settore delle raccolte storicoartistiche cittadine, ${ }^{53}$ Visconti svolgerà tenacemente e per un lungo arco di anni un lavoro di cernita, raccolta e acquisizione di materiali, favorendo equamente le istituzioni storico-artistiche cittadine attraverso $i$ cui cataloghi si muoverà con confidenza e competenza crescenti. Legittimato dall'autorità degli uffici assunti, arriverà anche a proporre, mosso da esigenze conservative e giustificato da logiche di pertinenza, il trasferimento di oggetti dall'una all'altra. ${ }^{54}$ La natura di queste considerazioni mostrano fecondo lo studio della sua figura di conservatore quando non di collezionista. ${ }^{55}$

\section{5. «Cosa DOBbIAMO DUNQUE FARE DEL CASTELlO?» (1886-1894)}

Nel 1886, quando, scongiurata la demolizione, per il castello di Milano si tratta di perfezionare il progetto di restauro, Visconti è dunque l'interlocutore istituzionale, come assessore alla pubblica istruzione e soprintendente scolastico ma è anche all'apice della sua attività di conservatore come presidente delegato del Museo Patrio di Archeologia, presidente della commissione amministratrice del Museo Artistico, e membro della commissione ordinatrice per il Museo del Risorgimento.

52 AVSV, cas. 115, fasc. 1871. Già nel febbraio del 1871 Visconti è incaricato dell'ordinamento del legato del medagliere Taverna.

53 Alla morte del conte Paolo Taverna, il 20 luglio 1871, Visconti ne prende il posto nella commissione incaricata dell'ordinamento di archivio storico, musei e biblioteche. L'incarico risale al 23 febbraio 1871, in AVSV, cas. 115.

54 Emblematica, al proposito, la richiesta di depositare il quadro raffigurante la Zecca di Milano al Gabinetto Numismatico ove avrebbe costituito valido documento di contesto al medagliere, plausibile alternativa quindi all'ostensione nella pinacoteca del Museo Artistico dove non vi era spazio per la grande tela, cfr. La Guadia 1989, nn. 1136, 1164, 1174.

55 Oltre all'apprezzata raccolta di materiali preistorici, Visconti radunò oggetti che documentano una varietà di interessi sconfinante nel mondo dell'artigianato (come, ad esempio, i bacili da barba tuttora esposti nel castello di Somma), del documento storico, della bibliofilia. Le poche ma accorate parole di cordoglio espresse nel giorno della morte dall'assessore anziano, Emanuele Greppi, ricordano anche «una magnifica e ricchissima collezione di carte geografiche della vecchia Milano», Atti del Municipio di Milano, (1910-1911), I, 502: Seduta del 30 maggio 1911. 
A questa data il dialogo con Beltrami è serrato e sebbene i frequenti incontri abbiano dissipato le loro parole, restano alcune lettere che l'architetto usava scrivere quando era fuori città o prossimo ad esserlo. $\mathrm{E}$ ' in una di queste circostanze che scrive non solo al collega e assessore ma all'amico per anticipare alcune delle importanti novità che riguardano il castello. ${ }^{56}$ Spiega che il Governo ha richiesto un rapporto contenente le clausole da includere nel contratto di formale cessione dell'edificio al Municipio, che la Commissione conservatrice ha quindi nominato una sotto-commissione, che egli stesso, con altri colleghi, vi è stato incluso; in sostanza lo rassicura scrivendo che il lavoro della Commissione non si è spinto a dare «imposizioni di restauri» che saranno piuttosto da «discutere a norma delle circostanze». Nella lettera, che illustra a Visconti i contenuti della nota relazione presentata da Celeste Clericetti precisandone le circostanze ${ }^{57}$ riferisce le clausole da inserire nel contratto concordate in commissione: nessun ostacolo alla demolizione della ghirlanda, sgombro di tutte le costruzioni aggiunte al concetto primitivo, riadattamento dei locali secondo le disposizioni primitive.

Il progetto di restauro si doveva ancora definire: puntualizzando le scelte artistiche solo parzialmente configurate nei disegni approntati nel 1884 per includervi quell'interpretazione 'civile' del monumento che andava maturando, perfezionando gli aspetti pratici (gli oneri finanziari e le tempistiche) e, soprattutto, indicando la destinazione d'uso da assegnare all'edificio. Queste scelte si preciseranno mentre si percorre il complesso iter di cessione dell'immobile al Comune, si implementano il rilievo e lo studio dell'edificio, si iniziano le prime demolizioni selettive..$^{58} \mathrm{Il}$ ruolo di Visconti è già emerso proprio in questa fase: a scongiurare, in particolare, il pericolo di adattare il castello a nuova sede del museo civico (1888). ${ }^{59}$

Nel novembre del 1889, però, Visconti decade dalla carica di assessore lasciando il compito a Giovanni Celoria. ${ }^{60}$ E' probabilmente

56 In appendice doc. 3.

57 Di Biase 1997, 50-51. Per il testo della relazione, datata 28 giugno 1886, cfr. Raccolta Beltrami, C III, 25.

58 Di Biase 1997, 51-53. Per il fondamento storico del progetto, cfr. Bellini 1997a, 10-11; 17-20.

59 Di Biase 1997, 49.

60 Su questo cordiale 'passaggio di consegne' si veda in Appendice doc. 13. Un commento in rapporto alla contemporanea e connessa questione del Museo di Storia Naturale in Grisoni, 2012, 101. 
nell'occasione di questo avvicendamento che, costretto dal risultato elettorale a lasciare, tra le altre cose anche la delega alla presidenza della Consulta (da cui deriva la conduzione del Museo Patrio di archeologia tra il 1884 e il 1889), ${ }^{61}$ compila un lungo memoriale, una cronologia di quanto si è fatto per il Castello dal 18 marzo 1887 al 21 giugno 1889. Scritto nelle finissima, inconfondibile grafia del marchese, compilato con una precisione ossessiva nella resa di particolari, dettagli, date, numeri di protocollo precisa ruoli, circostanze e compiti personalmente assunti nell'estenuante e non ancora compiuta storia di questo grande progetto in cui il restauro dell'architettura si vuole saldamente congiungere alla funzione che essa dovrà assolvere. Al documento si trova unita una grata lettera di Giovanni Celoria che, restituendo il plico (agosto 1894) ammette:

poco potei conchiudere, ma se gli assessori passano, resta il Castello e restano gli uomini che ad esso dedicarono studii e cure; fra essi lei. Con tali uomini il Castello trionferà di tutti gli ostacoli che passioni o verità di parte potranno creare. ${ }^{62}$

Il memoriale riflette, con particolare riguardo alla tutela del Castello, quella vitalità che è già stata assegnata all'assessore Visconti nel lavoro svolto in Consulta anche per il suo rilancio. ${ }^{63}$ L'uscita dalla Giunta tuttavia non frenerà l'intraprendenza del marchese che lasciata la Consulta del Museo Patrio conserverà i propri compiti al Museo Artistico - seguendo in particolare con Guido Cagnola il confluirvi del patrimonio della Società del Museo d'Arte industriale ceduto al Comune $^{64}$ - e del Risorgimento. Le due istituzioni ancora coabitano nei

${ }^{61} \mathrm{La}$ Guardia 1989, n. 2389/1-3 per il Verbale di adunanza del 16 novembre 1889 in cui Visconti saluti i colleghi. Per le lettere di ringraziamento, una firmata da tutti i membri della Consulta (Emilio Belgiojoso, Emilio Visconti Venosta, Luca Beltrami, Felice Calvi, Cesare Cantù, Antonio Caimi, Gustavo Frizzoni, Emilio Seletti) e l'altra del solo segretario Giulio Carotti, disposte nella seduta del 4 marzo 1890, ivi, n. 2393/1-3. Le minute di risposta si ritrovano in AVSV, cas. 116, fasc. 1890. Visconti rientrerà in Consulta nel 1895 a sostituire il defunto Cesare Cantù, AVSV, cas. 116, fasc. 1895, Lettera del 6 maggio 1895.

${ }^{62}$ In appendice doc. 31. Il Memoriale, manoscritto in grafia di Carlo Ermes Visconti, consta di fogli ripiegati e legati a formare un quaderno di 26 pagine scritte.

63 La Guardia 2012, 366.

64 AVSV, cas. 115, fasc. 1889. 
pochi locali del Salone ai Giardini Pubblici la cui demolizione è però inevitabile ora che vi si addossa l'erigendo nuovo palazzo del Museo Civico, inaugurato nell'aprile del 1892. Così, quando il successivo 30 giugno, il Regio Commissario straordinario convoca la commissione speciale istituita pochi giorni prima dalla Giunta Comunale per studiare l'uso più opportuno del castello di Milano, riattivando gli studi sull'edificio oramai pressati da più fronti, Visconti non solo è tra i convocati ma è designato a presiederla, in qualità di conservatore del Museo artistico municipale e della Scuola Superiore d'arte ma anche di membro della Commissione del Museo del Risorgimento. ${ }^{65}$

Dalla minuta di risposta trapelano entusiasmo, gratitudine e compiacimento perché, risponde, si tratta di

un progetto che accarezzo da parecchi anni e che ha subito nel mio pensiero tutte le fasi delle cose fortemente amate e desiderate. ${ }^{66}$

Dopo avere inizialmente rifiutato di presiederla, desiderando per sé la libertà di esprimersi libero dai vincoli che il ruolo avrebbe potuto imporre, poi accetterà assolvendo abilmente proprio questo compito.

La commissione inizia i propri lavori, dopo l'estate, il 15 novembre, riunendosi nell'ufficio del sindaco, ora Giuseppe Vigoni. ${ }^{67} \mathrm{La}$ domenica seguente, giorno 20, i commissari visitano i locali del castello, muniti di uno speciale lasciapassare fornito dall'Autorità militare che ancora dispone dei locali. ${ }^{68}$ Nei giorni successivi si acquisiscono i rilievi dell'intero edificio che sono custoditi dall'ufficio Regionale. ${ }^{69}$ Visconti dirige con precisione le operazioni, vigila sul passaggio di queste carte, spesso semplicemente siglandole. E' assistito infatti dal segretario comunale, Giuseppe Luini, le cui mansioni si estendono alla commissione per il castello. Compare inoltre anche Gaetano Moretti, scrupoloso alter ego di Beltrami all'Ufficio Regionale. Questi, già deputato del Parlamento, si trattiene per alcuni brevi periodi a Roma; dunque i lavori della commissione non si arrestano, anzi accelerano, nelle vacanze natalizie quando Beltrami, rientrato a Milano, personalmente avvisa

\footnotetext{
${ }^{65}$ In appendice doc. 14.

66 In appendice doc. 14.

67 In appendice doc. 16

68 In appendice doc. 17

${ }^{69}$ In appendice doc. 18 e doc. 19.
} 
Visconti perché ne approfitti per convocare la commissione. ${ }^{70}$ L'amico presidente risponde con una sollecita convocazione a palazzo Marino, nella sala della Soprintendenza scolastica, per il 3 di gennaio del $1893 .{ }^{71}$ Il 5 gennaio Beltrami, nuovamente in partenza per Roma, chiede a Visconti di incontrarsi, includendo nella ristretta riunione anche l'architetto Arcaini cui, scrive, devono darsi istruzioni. ${ }^{72}$

Gli incontri e i sopralluoghi al castello si svolsero dunque a ritmo piuttosto serrato in quei mesi: per rilevare le misure dei locali e verificarne la praticabilità, per valutare le opere da eseguirsi ma anche per discutere e contenere la 'ridda' delle possibili destinazioni ${ }^{73}$ anche se, come noto, nemmeno questa volta si giungerà ad una definitiva assegnazione. ${ }^{74}$

Sul riuso del castello si ritrovano alcuni appunti nell'archivio del marchese. Perfettamente convergenti agli intenti del progettista, permettono di qualificare l'apporto di Visconti come esponenziale all'obbiettivo perseguito dal gruppo, tenace nella funzione di coordinamento. ${ }^{75}$

Visconti infatti si identifica in quel «manipolo piccolo, pur troppo, ma compatto di amanti delle memorie patrie» che da lungo tempo perseguono il desiderio della cessione del castello al municipio. E' testimone dei fatti che è in grado di rievocare ripercorrendo dettagliatamente le pregresse proposte di riuso: quella di accogliere uffici pubblici o la sede comunale (naufragata esaminando la posizione dell'edificio, «alquanto eccentrica», e la fisica disposizione degli ambienti, «enormi ed illuminati da spaziosissime finestre ma poco numerose»), ma anche quella, «fortunatamente» abbandonata, di collocarvi il museo di storia naturale. E' conquistato dalla particolarità del caso che offre l'opportunità di riscoprire il valore storico del monumento e di attualizzarne il significato ed il ruolo per i milanesi. E' dunque consapevole delle novità insite nell'approccio di restauro che, tradotto in parole sue, consiste nella volontà di esibire al pubblico non solo l'esterno ma anche «gli arti-

70 In appendice doc. 21.

71 In appendice doc. 22.

72 In appendice doc. 23.

73 In appendice doc. 18.

74 cfr. Di Biase 1997, 50.

75 Si tratta di dieci fogli sciolti manoscritti al recto in grafia di Visconti, muti al verso. Conservati in AVSV, cass. CC.. Ad essi si riferiscono i virgolettati del titolo e $\mathrm{i}$ seguenti nel testo. 
stici particolari dei suoi ambienti» esaltando il ruolo simbolico del monumento e assimilando dunque l'idea che esso non possa che accogliere, per rigida coerenza, solo funzioni strumentali allo scopo, rispondendo così alla domanda che si era posto, all'inizio del suo discorso, sul destino del castello. Inizia per questo una rassegna che passando attraverso la Pinacoteca, la Biblioteca e il Gabinetto numismatico, quindi la Pinacoteca Ambrosiana e la Fondazione artistica Poldi-Pezzoli (realtà che, per ragioni diverse, hanno già la loro sede opportuna) circoscrive il gruppo delle quattro istituzioni che sono espressione della cultura storica artistica cittadina: l'Archivio storico municipale, il Museo patrio di archeologia, il Museo municipale d'arti e il Museo del Risorgimento nazionale. Quindi descrive lo stato di quei luoghi, mostrando di conoscere a fondo la consistenza delle collezioni e, per il Museo Artistico e del Risorgimento in particolare, anche dell'architettura che li ospita. La relazione si interrompe in questa disamina che giunge alla conclusione di dovere assegnare a questi quattro istituti - cui si aggiungeranno la Società Storica Lombarda (depositaria dei medesimi valori) e la Scuola d'arte applicata all'industria (inscindibilmente legata al Museo Artistico e testimone della finalità didattica connessa allo studio della storia e dell'arte) - le sale restaurate del castello. Il manoscritto non è datato ma taluni riferimenti inclusi nel testo consentono di trattenerlo entro i primi mesi del 1893 e di ricondurlo ad un discorso tenuto in una qualche seduta della Commissione o forse, per la pungente ironia che lo vivacizza, destinato a stuzzicare con brio l'opinione pubblica. ${ }^{76}$

Nel frattempo, sincronizzati sulla presenza in città di Beltrami, gli incontri rivelano i nomi dei collaboratori e quello di Ranieri Arcaini, in particolare, l'architetto cui è delegato il compito di seguire i rilievi e la protezione delle opere di demolizione. Iniziano infatti i primi lavori così, nel mese di gennaio del 1893 l'autorità militare concede ad uso della commissione il locale n. 54 (in realtà due locali, uno grande e uno piccolo) che è situato al piano terreno del gran cortile, a sinistra, entrando. ${ }^{77}$ Il 31 di quello stesso mese Arcaini informa il presidente, Visconti, che occorre imporre d'ufficio all'impresa appaltatrice dei lavori «di risparmiare nelle demolizioni del Muraglione N-O la parte antica ora

76 Il documento annuncia per il successivo mese di maggio la definitiva cessione del castello al Comune. Il passaggio avverrà tra il 25 ottobre e il 9 novembre 1893.

77 In appendice doc. 26. 
scoperta, e che Ella ben conosce». ${ }^{78}$ L'allarmante lettera allude ai lavori di demolizione delle ghirlanda nel corso dei quali si trovano parte viscontee che si vogliono prudenzialmente risparmiare. Arcaini non è solo diligente ma anche indispettito; vorrebbe meno burocrazia, meno passaggi di carte, più autorità individuale in ragione del ruolo assunto di rappresentante dell'ufficio regionale. I contenziosi con l'impresa Bonomi sono noti; ${ }^{79}$ come è noto Arcaini, tanto per zelo che per puntiglio; ${ }^{80}$ la piccata lettera ne conferma il ruolo e le relative ricadute tanto sulla conduzioni dei lavori che nei rapporti con l'impresa, fin dall'esordio nel lungo cantiere di restauro del castello.

Il 19 febbraio 1894 Beltrami interpella Visconti. ${ }^{81}$ Sono in corso i lavori al torrione sud-est (come noto riconvertito a serbatoio per l'acquedotto cittadino). Si sta rimarginando la cortina esterna, riconfigurandone il profilo che viene elevato ad una maggiore altezza (anche in considerazione degli effetti che questo avrà nella ricomposizione del discusso fronte verso la città) e sagomandone la cresta con il disegno di una merlatura di coronamento. L'architetto chiede a Visconti di «esaminare da vicino i lavori della merlatura del torrione»: vuole che «si provveda a fare un lavoro un poco più decente» ed è certo che la voce dell'amico sarà più convincente della sua presso il sindaco Vigoni. ${ }^{82}$

Nell'autunno del 1894, finalmente smantellati i padiglioni dell'Esposizione Nazionale, ${ }^{83}$ i lavori riprendono e lo studioso tedesco Paul Müller-Walde prosegue gli 'assaggi' delle murature della Sala della Torre e di quella del Tesoro. I 'rinvenimenti' sono di eccezionale importanza. Beltrami ne parla a Visconti in due lettere. La prima, datata 28 novembre 1894, è la conferma dell'esistenza di pitture nella Sala della

78 In appendice doc. 28.

79 Di Biase 1997, 51-52.

$80 \mathrm{Si}$ allude all'apporto, tanto in fase di progetto che di conduzione dei lavori, per la costruzione della torre Umberto I, cfr. Raccolta Beltrami, A IV, 5. Per il ridondante riconoscimento dei suoi meriti si veda anche Raccolta Beltrami, Op. 315.

${ }^{81}$ In appendice doc. 30.

82 Sulle interferenze, ostative, di Vigoni e sul suo 'ravvedimento' cfr. Di Biase 1997, 53.

${ }^{83}$ Visconti rifiutò la proposta di presiederne il comitato promotore, in AVSV, cas. 116, fasc. 1894. Si è portati a credere che sia una manifestazione di contrarietà alla sede scelta per l'allestimento dei padiglioni, notoriamente avversata radicalmente da Beltrami e quindi circostanza di ulteriore solidarietà tra i due. 
Torre ove appare «il principio della decorazione della volta costituito da grossi tronchi d'albero ${ }^{84}$ (Fig. 1). La seconda, che giunge, con gli auguri, il 26 dicembre successivo, è per condividere l'inaspettata comparsa di «tracce importanti di pitture nella Sala del Tesoro (Rocchetta)» che nell'immediato giudica «anteriori a Lodovico il Moro - maniera di V. Foppa» ${ }^{85}$ (Fig. 2).

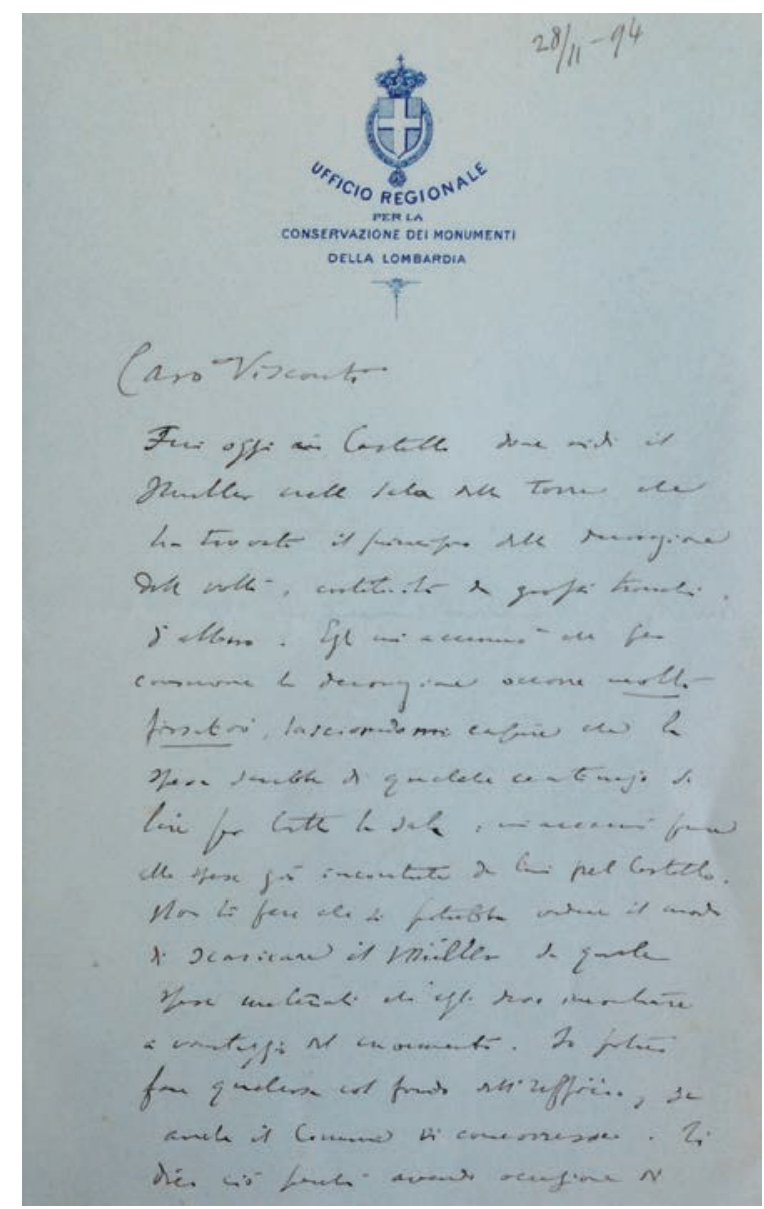

Fig. 1.

${ }^{84}$ In appendice doc. 34.

85 In appendice doc. 35. 


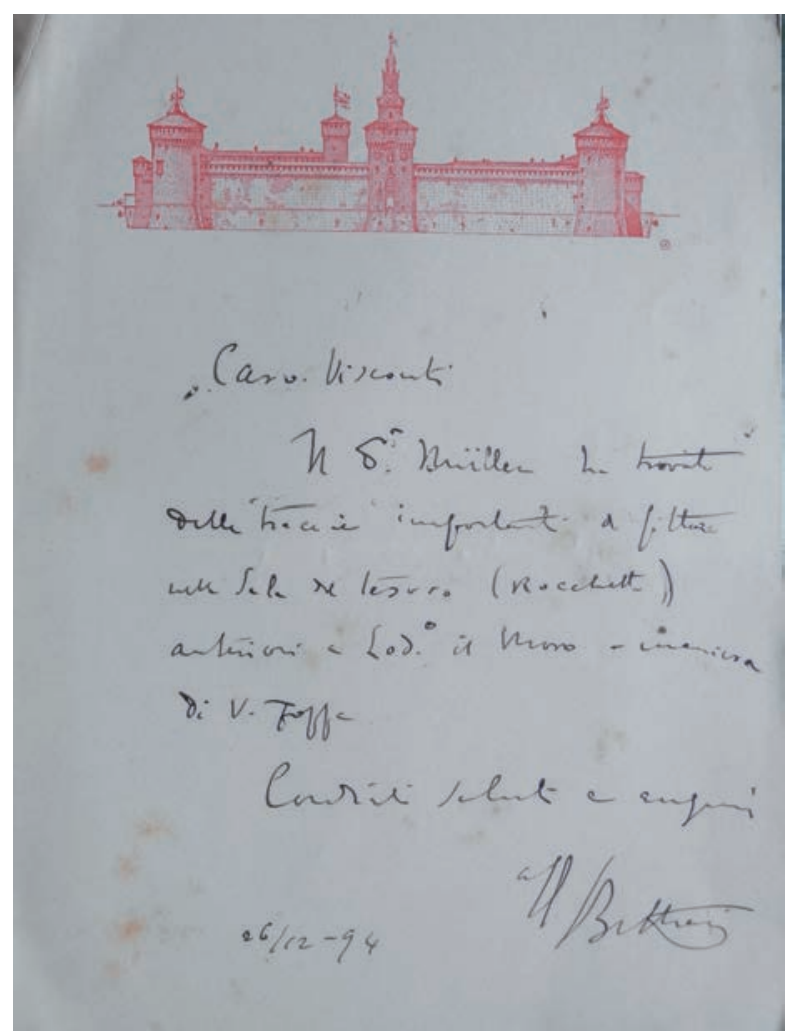

Fig. 2.

Mentre dunque condivide con l'amico le importanti novità e confida le proprie impressioni sulla maniera e l'epoca delle pitture emerse, manifesta anche preoccupazioni di carattere tecnico e d'ordine pratico in merito alla loro conservazione. Beltrami infatti avverte Visconti che, soprattutto nella Sala della Torre (la Sala delle Asse), a detta di MüllerWalde, occorre «molto fissativo»; il che si traduce, pragmaticamente, in «qualche centinajo di lire per tutta la sala». Chiede quindi un sostegno, di natura economica, «per scaricare il Müller da quelle spese materiali» che, aggiunge, in parte ha già sostenute.

Sulla Sala delle Asse persistono tuttora molti dubbi determinati dalla frammentarietà delle fonti finora rintracciate. La questione non riguarda solo la realizzazione dell'opera ma anche la sua durata nel tempo e soprattutto la fase tardo ottocentesca di riscoperta del linguag- 
gio leonardesco e bramantesco e di restauro delle pitture superstiti. ${ }^{86}$ Tra il 1893 e il 1895 la descrizione di quanto riemergeva dai fortunati assaggi di Müller Walde fu come noto condizionata dal riserbo con cui, a tutela della paternità della scoperta, si volle diffondere la notizia, soprattutto all'interno della comunità scientifica ${ }^{87} \mathrm{E}$ ' stato osservato che deriva probabilmente da questa 'riserva' l'inaspettata carenza di materiale iconografico e di precise descrizioni; così come pare che pochi ebbero accesso ai quei luoghi prima dei restauri dove, peraltro, si immagina difficile la lettura di una decorazione affiorante a frammenti al di sotto di sovrapposti strati di finitura. Le inedite espressioni utilizzate da Beltrami per descrivere a Visconti quanto visto sembrano ora dimostrarlo; soprattutto se poste a confronto con le sue successive descrizioni e pur tenendo conto del contesto in cui furono amichevolmente espresse e della immediatezza della suggestione.

Nell'attesa di capire non tanto se Visconti, avutane notizia, abbia visitato le sale (cosa peraltro assai probabile) ma, soprattutto se ne abbia in qualche modo trattenuto un ricordo (cosa assai più utile), le lettere finora rintracciate sollevano piuttosto un interrogativo relativo ai lavori precauzionali da condursi. Lo stato di conservazione evidentemente non era buono perché la precisazione che occorresse 'molto' fissativo, soprattutto nella sala delle Asse, sia che la si consideri in rapporto alla vastità della superficie che alla consistenza del degrado, comunque denuncia l'importanza del lavoro urgente da compiersi. La preoccupazione per le spese già affrontate, oltre a quelle da affrontarsi, impongono di valutare anche quale fu il limite dato alla rimozione dei sovrapposti strati di finitura e al consolidamento preventivo dei sottostanti e di considerare per questo plausibile l'ipotesi che, almeno in alcune parti, vi siano stati interventi preventivi precedenti all'opera di Ernesto Rusca. Conducono anche in questa direzione le più recenti acquisizioni d'archivio ${ }^{88} \mathrm{Si}$ tratta di questioni di carattere tecnico che riguardano le procedure di restauro e che esulano, fatta esclusione delle ricadute economiche, le questioni che più direttamente sembrò affrontare Visconti.

86 Si veda Fiorio 2006 e Fiorio, Lucchini 2007.

87 Tali cautele sono dettagliatamente illustrate da Beltrami stesso in Beltrami 1897a e Beltrami 1897b. Per le minute di entrambi questi due articoli, Raccolta Beltrami, C III, 27.

88 Catturini 2013. 


\section{VERSO L'APERTURA DEI MUSEI DEL CASTELLO (1895-1900)}

Nei successivi cinque anni è facile invece documentarne il ruolo nel trasporto e nell'ordinamento dei musei al castello; poiché davvero molte, e puntuali, sono le testimonianze. Nel 1896 il Museo del Risorgimento si trasferisce al piano primo del cortile della Rocchetta i cui ambienti di fatto aveva già opzionati in occasione dell'Esposizione Nazionale dell'anno precedente; Visconti è chiamato ad occuparsene..$^{89}$ Nell'estate del 1897 si iniziano a muovere alcuni materiali del Museo Patrio; egli è in corrispondenza per questo con Giulio Carotti, segretario di Brera e, per regolamento, anche del museo. ${ }^{90}$ Nel giorno della consegna della medaglia d'oro a Beltrami 'La Perseveranza' annoterà i nomi e renderà lodi a coloro che lavorarono all'ordinamento: per il museo patrio Emilio Seletti, Alfonso Garovaglio, Gustavo Frizzoni, Luca Beltrami e Giulio Carotti; per il Museo artistico Giovanni Battista Vittadini, Giulio Pisa e Carlo Bazzero. Il nome di Visconti è l'unico che figura per entrambi. ${ }^{91} \mathrm{~A}$ sostegno dell'amico, Giuseppe Vigoni vorrà che sia anche scritto che

negli ultimi cinque anni, sempre lavorando nel silenzio, che ne rispecchia la modestia, dedicandovi studio, fatiche e tutta la sua speciale competenza, compì il trasporto nel Castello e l'ordinamento artistico-scientifico della numerosa e preziosa raccolta. ${ }^{92}$

Nel corso di questa aggregazione la gestione del catalogo si era complicata. Occorreva smontare e rimontare gli insiemi architettonici, radunare materiali di una medesima collezione, magari di un medesimo museo, sparsi in più sedi o ricoverate nei depositi. Bisognava inoltre decidere la disposizione degli oggetti, definire i percorsi, congiungere le collezioni generatrici delle diverse raccolte in un museo le cui sale dovevano ancora essere completate e restaurate.

Beltrami descriverà molto bene la complessità e la delicatezza della questione in un lungo esaustivo resoconto in cui il nome di Visconti, oramai deceduto, ricorre più volte; non solo ad indicarne il noto supporto garantito all'architetto per «far prevalere il concetto di

\footnotetext{
89 AVSV, cas. 116, fasc. 1896.

90 Cfr., ad esempio, La Guardia, nn. 2640 e 2642.

${ }^{91}$ La Perseveranza, 18 dicembre 1900.

92 Lettera di Giuseppe Vigoni alla redazione, La Perseveranza, 18 dicembre 1900.
} 
una razionale utilizzazione del castello», ma anche a ricordarne, con i nomi dei consultori Biondelli, Ceriani, Cantù, Mongeri, Bertini, Brocca, Carlo ed Emilio Belgiojoso, Calvi, Carcano, Caimi, Garovaglio, i risultati raggiunti dalla Consulta del Museo Patrio «nel momento in cui il rinnovamento edilizio di Milano disperdeva tante memorie storiche ed artistiche». Infine a precisare che la compilazione dello schedario degli oggetti del Museo Artistico era stata sua «precipua cura», affermando quindi a chiare lettere che

il ritiro del marchese Carlo Ermes Visconti, conseguente dall'insediamento dell'Amministrazione Mussi, aveva privato i due musei che si erano compenetrati nelle sale della Corte Ducale, di quella lunga pratica ed esperienza, che il Visconti aveva accumulato in un trentennio di assidua prestazione personale a vantaggio del patrimonio artistico del Comune. ${ }^{93}$

A gennaio del 1900 Visconti aveva infatti dovuto lasciare la direzione del Museo. ${ }^{94}$ Questa circostanza, seguita dalle spiacevoli accuse mosse anche nei suoi confronti nell'ambito dell'inchiesta municipale avviata per verificare presunti ammanchi nel patrimonio artistico comunale, ${ }^{95}$ ne determineranno il duro distacco, persistente anche dopo il chiarimento, ${ }^{96}$ dagli ambienti della politica culturale cittadina. ${ }^{97}$

93 Beltrami [1912].

94 Rassegna le proprie dimissioni il 21 dicembre 1899. Sul passaggio di consegne si veda ora Basso 2012. Per il rinnovo delle cariche direttive cfr. anche Atti del Municipio di Milano, (1899-1900), I, 292: Seduta del 3 luglio 1900.

95 Atti del Municipio di Milano, (1904-1905), I, 250: Seduta del 18 aprile 1905 per la consegna del cosiddetto plico Sinigaglia e ivi, 287-294: Seduta del 28 aprile 1905, per l'interrogazione di Ernesto Bazzero e, soprattutto, per le parole di Beltrami e Gabba in sua difesa.

96 Per la discussione conclusiva e i risultati dell'inchiesta che «consacrano ancora una volta la fama della illibata onestà e della diligenza dei benemeriti cittadini cui era commessa la custodia delle collezioni comunali e di quelle di proprietà dello Stato affidate al Comune», Atti del Municipio di Milano, (1905-1906), I, 223-233: Seduta del 13 febbraio 1906 e ivi, II, IX.

97 Informato della decisione presa dal Consiglio Comunale nella seduta del 11 aprile 1905 con cui lo si nomina membro del Consiglio direttivo della Galleria d'Arte Moderna, Visconti rifiuta. Più d'una le minute di risposta allegate alla lettera di nomina: esternano gratitudine «per coloro che mi hanno dato il loro suffragio» e riconoscenza «per chi ha pensato a questo atto di riparazione oggi però ancora prematuro», dimostrando, con diverse gradazioni di stizza, la ferma volontà di estraniarsi dagli incarichi municipali nel mutato scenario politico, AVSV, cas. 116, fasc. 1905. 
Non può non rilevarsi, però, che in questa ultima fase la corrispondenza tra Visconti e Beltrami tende ad attenuarsi. Pur considerandosi una fatale dispersione delle carte o, all'inverso, un infittirsi degli incontri, si deve ricordare il fatto oggettivo che in quegli stessi ultimi cinque anni, la loro amicizia, era stata messa alla prova dagli esiti del concorso di secondo grado per il completamento della facciata del Duomo.

\section{RINGRAZIAMENTI}

Il presente lavoro è parte di un più estensivo esame dell'epistolario di Carlo Ermes Visconti che si conserva presso il Castello Visconti di San Vito di Somma Lombardo, proprietà della Fondazione Visconti di San Vito. Devo profonda gratitudine al presidente della Fondazione stessa, avvocato Gaetano Galeone, che ha permesso lo studio di queste carte.

\section{BIBLIOGRAFIA}

AFD, VII. Annali Fabbrica del Duomo di Milano, VII (1885).

Amadini 2013. P. Amadini, Arti dell' Asia Orientale tra pubblico e privato: due raccolte esemplari. Dal 1870 cent'anni di collezionismo cinese e giapponese a Milano, Tesi di dottorato, Ca' Foscari, Venezia, 2013.

Atti I 1880. Atti della Commissione Conservatrice dei monumenti e oggetti d'arte e d'antichità nella provincia di Milano. Puntata prima che abbraccia il periodo dall'istituzione a tutto il luglio del 1880 annessi al volume VII dell'archivio storico lombardo, ASL, 7 (1880).

Atti II 1881. Atti della Commissione Conservatrice dei monumenti e oggetti d'arte e di antichità nella provincia di Milano. Puntata seconda in cui si comprendono le discussioni dall'agosto del 1880 al luglio del 1881 annessa al volume VIII dell'Archivio Storico Lombardo, ASL, 8 (1881).

Atti parlamentari, 1900. Atti parlamentari, Senato del Regno, Discussioni, 1900-1902, 197, 22 novembre 1900.

Barbiera 1901. R. Barbiera, Il salotto della contessa Maffei e Camillo Cavour, Milano, Fratelli Treves, 1901.

Basso 2012. L. Basso, 18 gennaio 1900. Un documento e qualche nota per il museo artistico municipale, in Libri \& Documenti, 38 (2012), 117-131.

Basso 2014. L. Basso, Insieme a Luca Beltrami per i musei del Castello Sforzesco: Giulio Carotti, Emilio Seletti, Carlo Ermes Visconti, in Luca Beltrami (1854-1933). Storia, arte e architettura a Milano, Milano, Castello Sforzesco, 27 marzo - 29 giugno 2014, Catalogo della mostra a cura di Silvia Paoli, Cinisello Balsamo, Silvana editoriale, 2014, 169-189. 
Bellini 1997a. A. Bellini, Luca Beltrami, tesi sul restauro: l'esempio del Castello di Milano, in Luca Beltrami e il restauro dei castelli, 1893-1993. Nel centenario dell'acquisizione del Castello da parte del Comune, Quaderni della Sezione Lombardia dell'Istituto Italiano dei Castelli, 7 (1997), 9-20.

Bellini 1997b. A. Bellini, Luca Beltrami architetto restauratore, in Luca Beltrami architetto. Milano tra Ottocento e Novecento, Triennale di Milano 10 novembre - 31 dicembre 1997, Catalogo della mostra a cura di Luciana Baldrighi, Electa, 1997, 92-140.

Bellini 2014. A. Bellini, Un borghese esemplare della Milano dell'Ottocento, in Luca Beltrami (1854-1933). Storia, arte e architettura a Milano, Milano, Castello Sforzesco, 27 marzo - 29 giugno 2014, Catalogo della mostra a cura di S. Paoli, Cinisello Balsamo, Silvana editoriale, 2014, 15-45.

Beltrami 1881. L. Beltrami, Il Lazzaretto di Milano, in G. Ottino, Milano e i suoi dintorni, Milano 1881, 263-266.

Beltrami 1882. L. Beltrami, Il Lazzaretto di Milano, ASL, 9 (1882), 403-441.

Beltrami, Forcella 1889. L. Beltrami, V. Forcella, Di alcune lapidi e frammenti di scultura recentemente trovati in Milano, in ASL, 15 (1889), 951-965.

Beltrami 1897a. L. Beltrami, L., Il Mercurio del Castello di Milano, I, La Perseveranza, 25-26 dicembre 1897.

Beltrami 1897b. L. Beltrami, L., Il Mercurio del Castello di Milano, I, La Perseveranza, 30-31 dicembre 1897.

Beltrami [1912]. L. Beltrami, La sistemazione dei musei nel castello sforzesco nel ventennio 1892-1911, in Raccolta Beltrami, B II, 69.

Boffi 1886. L. Boffi, Il palazzo Vitelleschi in Corneto Tarquinia, Hoepli, Milano, 1886.

Boriani 1992. M. Boriani, «Il popolo intende ed ama naturalmente le antiche memorie purchè le sieno evidenti». Uso e abuso dei monumenti nella costruzione della Milano ottocentesca, in La Milano del Piano Beruto (1884-1889). Società, urbanistica e architettura nella seconda metà dell'Ottocento, I, a cura di R. Rozzi, Milano, Guerini, 1992, 385-404.

Boriani, Rossari 1992. M. Boriani, A. Rossari, Urbanistica milanese nella seconda metà dell'Ottocento. Tra 'savia economia', 'giuste esigenze dell'arte' e 'insormontabili ostacoli nella mania di guadagno', in La Milano del piano Beruto (1884-1889). Società, urbanistica e architettura nella seconda metà dell'Ottocento, II, a cura di M. Boriani, A. Rossari, Milano, Guerini, 1992, 1-76.

Bortolotto, Massari 1992. Bortolotto, S., Massari, G., I monumenti e la città. Cronologia delle fonti ufficiali, in La Milano del Piano Beruto (1884-1889). Società, urbanistica e architettura nella seconda metà dell'Ottocento, I, a cura di R. Rozzi, Milano, Guerini, 1992, 428-431.

Casati 1876. C. Casati, Vicende edilizie del castello di Milano, Milano, Libreria editrice Brigola, 1876.

Catturini 2013. C. Catturini, La Sala della Asse di Luca Beltrami: alcune novità documentarie sull'attività di Ernesto Rusca decoratore e restauratore, con qualche nota sull'allestimento di questo ambiente nella prima metà del Novecento, Rassegna di Studi e Notizie, 40, 36 (2013), 63-76. 
Costa 2006. P. Costa, The Sala delle Asse in the Sforza Castle in Milan, tesi Ph.D. Pittsburgh University, Pittsburgh 2006.

Di Biase 1997. C. Di Biase, Luca Beltrami e il progetto per il Castello Sforzesco di Milano. Note sul metodo e sul cantiere di restauro, in Luca Beltrami e il restauro dei castelli, 1893-1993. Nel centenario dell'acquisizione del Castello da parte del Comune, Quaderni della Sezione Lombardia dell'Istituto Italiano dei Castelli, 7 (1997), 35-83.

Favole 1969. P. Favole, Boffi, Luigi, in DBI, (11) 1969, 166-167.

Finocchi 1978. L. Finocchi, Edilizia scolastica a Milano dal 1860 al 1885, Storia Urbana, II, 6, (1978), 85-129.

Fiorio 2006. M. T. Fiorio, 'Infra le fessure delle pietre': la sala delle Asse al castello Sforzesco, in Il Codice di Leonardo da Vinci nel Castello Sforzesco, catalogo della mostra (Milano, Castello Sforzesco, 24 marzo - 21 maggio 2006) a cura di P. C. Marani, G.M. Piazza, Milano, Electa, 2006, 21-29.

Fiorio 2007. M.T. Fiorio, A. Lucchini, Nella Sala delle Asse, sulle tracce di Leonardo, Raccolta Vinciana, 32 (2007), 101-140.

Fratelli 2012. M. Fratelli, Dal legato Marchesi Fogliani all'istituzione della Galleria d'Arte Moderna, in Musei nell'Ottocento. Alle origini delle collezioni pubbliche lombarde. Giornate di studio (Milano, Palazzo Moriggia, 7 - 8 ottobre 2010), a cura di M. Fratelli, F. Valli, Torino, Allemandi, 2012, 391-410.

Gazzetta 1893. Gazzetta Ufficiale del Regno d'Italia, 94 (1893).

Giustina 1991. I. Giustina, Istruzione, cultura, assistenza. La nuova architettura pubblica della città, in Milano nell'Unita nazionale (1860-1898), a cura di G. Rumi, A.C. Buratti, A. Cova, Milano, Cariplo, 1991, 193-228.

Grifoni 1992. P. Grifoni, Gli operatori, in Bencivenni, M., Dalla Negra, R., Grifoni, P., Monumenti e istituzioni, II,Alinea, Firenze, 1992, 278-312.

Grisoni 2008. M.M. Grisoni, Carlo Ermes Visconti: tra privato collezionismo e tutela dei monumenti patri, Rivista della Società Storica Varesina, 25 (2008), 117-158.

Grisoni 2012. M.M. Grisoni, Carlo Ermes Visconti e la cultura tecnico scientifica milanese ottocentesca: appunti intorno al carteggio con Antonio Stoppani conservato nel Castello di Somma Lombardo, Rivista della Società Storica Varesina, 29 (2012), $85-108$.

Grisoni 2014a. M.M Grisoni, 1882-1892. Milano si progetta, in Il palazzo del Museo di Storia Naturale. Un'architettura della Milano Belle Époque, a cura di P. Livi, Milano, Silvana editoriale, 2014, 17-43.

Grisoni 2014b. M.M. Grisoni, Il Cipresso di Somma Lombardo: un precoce caso di tutela, Rivista della Società Storica Varesina, 31 (2014), 145-164.

Guida di Milano, ad annum Guida di Milano, ideata e compilata da G. Savallo, Milano, 1882-1900.

Jan 1863. G. Jan, Allocuzione del direttore Giorgio Jan pronunciata il giorno della solenne inaugurazione della nuova sede del Civico Museo (7 gingno 1863), Milano, Pirola, 1863.

Janiro 1979. N. Janiro, Celoria, Giovanni, in DBI, 23 (1979), 464-469.

La Guardia 1983. R. La Guardia, L'archivio privato di Pompeo Castelfranco nelle civiche 
raccolte archeologiche del Comune di Milano, Comune di Milano Settore cultura e spettacolo, Milano, 1983.

La Guardia 1989. R. La Guardia, L'archivio della Consulta del Museo Patrio di archeologia di Milano (1862-1903), Comune di Milano Settore cultura e spettacolo, Milano 1989.

La Guardia 2012. R. La Guardia, I depositi delle collezioni del Gabinetto numismatico e del Museo Patrio di Archeologia di Brera a Castello Sforzesco nei fondi dell'archivio del CASVA - Centro di alti studi sulle arti visive del Comune di Milano, in Musei nell'Ottocento. Alle origini delle collezioni pubbliche lombarde. Giornate di studio (Milano, Palazzo Moriggia, 7-8 ottobre 2010), a cura di M. Fratelli, F. Valli, Torino, Allemandi, 2012, 355-369.

Mongeri 1878. G. Mongeri, Il nuovo museo artistico municipale, ASL, 5 (1878), 517 535.

Novati 1912. F. Novati, Atti della Società Storica Lombarda, ASL, 17 (1912), 198-202.

Occhipinti 2012. C. Occhipinti, Milano nei progetti dei giovani architetti civili, in Architettura a Milano negli anni dell'Unità, a cura di Maurizio Grandi, Milano, Libraccio 2012, 183-202.

Ricci 1997. G. Ricci, Luca Beltrami allievo e docente, in Luca Beltrami architetto. Milano tra Ottocento e Novecento, Triennale di Milano 10 novembre - 31 dicembre 1997, catalogo della mostra a cura di L. Baldrighi, Milano, Electa, 1997, 142-151.

Rossari 1997. A. Rossari, Luca Beltrami e l'urbanistica milanese tra Ottocento e Novecento, in Luca Beltrami architetto. Milano tra Ottocento e Novecento, Triennale di Milano 10 novembre - 31 dicembre 1997, Catalogo della mostra a cura di Luciana Baldrighi, Milano, Electa, 1997, 76-91.

Savare' 1994. G. Savare', La Commissione milanese (1877-1890), in Del restauro in Lombardia. Procedure, istituzioni, archivi 1861-1892, a cura di G. P. Treccani, Milano, Guerini, 1994, 235-266.

Treccani 1992. G. P. Treccani, Strumenti normativi e pratica della tutela monumentale, in La Milano del piano Beruto (1884-1889). Società, urbanistica e architettura nella seconda metà dell' Ottocento, vol. I, a cura di M. Boriani, A. Rossari, Milano, Guerini, 1992, 245-259.

Vercelloni 1991. V. Vercelloni, Le trasformazioni urbane, in Milano nell'Unita nazionale (1860-1898), a cura di G. Rumi, A.C. Buratti, A. Cova, Milano, Cariplo, 1991, 137-163.

Visconti 1880. C.E.V. [Carlo Ermes Visconti], Museo artistico municipale, in Gli istituti scientifici, letterari ed artistici di Milano, Milano, Pirola, 1880, 713-721.

Visconti Venosta 1904. G. Visconti Venosta, Ricordi di gioventù. Cose vedute o sapute. 1847-1860, Milano, Tipografia Cogliati, 1904.

Zatti 2012. P. Zatti, Un «visibilio di visioni». Torino 1884 e la nascita delle raccolte storiche milanesi, in Musei nell'Ottocento. Alle origini delle collezioni pubbliche lombarde. Giornate di studio (Milano, Palazzo Moriggia, 7-8 ottobre 2010), a cura di M. Fratelli, F. Valli, Torino, Allemandi, 2012, 281-287. 


\section{APPENDICE}

NotA. - L'elenco è cronologico. La numerazione progressiva è strumentale al richiamo dei documenti nel testo. I documenti si conservano presso l'Archivio Visconti di San Vito di Somma Lombardo (d'ora innanzi AVSV); nell'indicare la posizione d'archivio la fonte è pertanto ovunque sottintesa. Si omette inoltre la trascrizione, riducendola a poche righe riassuntive, per quelle lettere i cui contenuti appaiono perlopiù di circostanza o meno pertinenti al tema.

doc. 1 - s.d. ma 1886: ${ }^{1}$ Luca Beltrami a Carlo Ermes Visconti, cas. CC, fasc. 1886

Saluta e anticipa l'occasione di un prossimo incontro.

doc. 2 - s.d. ma 1886: ${ }^{2}$ Luca Beltrami a Carlo Ermes Visconti, ${ }^{3}$ cas. CC, fasc. 1886

Nel contesto di una nomina non esplicita nel testo, segnala i nomi, concordati con Boito e Pirovano, ${ }^{4}$ di G. Pagani, ${ }^{5}$ G. Landriani ${ }^{6}$ e G. Boffi.

doc. 3 - s.d. ma 1886: Luca Beltrami a Carlo Ermes Visconti, cas. CC, fasc. 1886

1 Per la lettera, non datata, si indica l'anno scritto in grafia dello stesso Visconti sulla camicia del mazzo in cui è stata rinvenuta all'atto della consultazione (aprile, 2011).

2 Idem

3 Su carta intestata 'Società degli Artisti e patriottica/Milano/via S. Giuseppe n. 4'.

4 Si tratta di Camillo Boito e Giuseppe Pirovano.

5 Verosimilmente Gentile Pagani.

6 Verosimilmente il fratello di Gaetano, Giuseppe, direttore de La Perseveranza.

7 Luigi Boffi (1846-1904), architetto formatosi alla Scuola Superiore di architettura di Brera, noto per alcuni progetti di ville partecipò, distinguendosi, non solo ad importanti concorsi cittadini ma anche di portata nazionale e internazionale (dal monumento alle Cinque Giornate alla facciata del Duomo fino al Monumento a Vittorio Emanuele II); cfr. Favole 1969. Il rilievo del palazzo Vitelleschi, cui allude Beltrami nella lettera, premiato dal Collegio degli ingegneri ed architetti di Torino, fu occasione, per l'autore, di comporre una breve monografia (Boffi 1886); le tavole, in particolare, apprezzate da Camillo Boito, divennero strumento didattico per gli allievi architetti di Brera; cfr. per questo, in particolare, Occhipinti 2012, 190. 


\title{
Caro Visconti
}

Ho ricevuto i due firmari e ti ringrazio in scritto per ora, riserbandomi di ringraziarti a voce appena che sarò di ritorno, fra quattro o cinque giorni, dal lago d'Orta dove mi reco domattina: verrò a bagolare un poco in ufficio. Riguardo alla notizia che mi chiedi ti dico intanto che il Governo ha sollecitato dalla C.C. una specie di rapporto contenente le clausole che si ritenessero necessarie per la Conservazione del Castello all'atto della cessione formale. La Comm. C. nominò una sottocommissione Clericetti, Castelfranco, Frizzoni, Guidini, Beltrami: ${ }^{8}$ io ho cercato di tenere le clausole nel senso più generico e semplice e cioè: nessun ostacolo demolizione ghirlanda - sgombro di tutte le costruzioni aggiunte al concetto primitivo - riadattamento dei locali secondo le disposizioni primitive man mano che si venisse a utilizzare detti locali. Il Clericetti relatore voleva perdersi in dettagli e in imposizioni di restauri che io credo prematuri e da discutere in seguito a norma delle circostanze. C'è stato da fare e sudare per conservare il rapporto ai giusti limiti senza toccare a chi spettasse la spesa dei restauri e in quali periodi si avessero a fare questi. Il cons. Zerbi mi disse poi che il Prefetto intendeva di metterci anche lui, a sua volta, lo zampino, ma non ne so nulla. In conclusione il compito della Sotto Commissione non è stato menomamente di fare studii di restauro. Ne parleremo più estesamente a voce, tuo aff. Luca Beltrami.

\section{doc. 4 - s.d. ma 1887 circa, ${ }^{9}$ Luca Beltrami a Carlo Ermes Visconti, cas. CC, fasc. Autografi \\ Accordi per la visita del campanile di S. Gottardo a Milano.}

\author{
doc. 5 - 1888, marzo 18: Luca Beltrami a Carlo Ermes Visconti, ${ }^{10}$ \\ cas. CC, fasc. 3
}

8 Si tratta della sotto-commissione nominata in seno alla Commissione Conservatrice (che scioglie le cifre C.C.) e composta, per l'esattezza, da Celeste Clericetti, Pompeo Castelfranco, Gustavo Frizzoni, Augusto Guidini, Luca Beltrami. Sui lavori della Commissione dedicata alla studio delle possibili destinazioni d'uso del castello cfr. Di Biase 1997, 49. Sulla cosiddetta 'relazione Clericetti' in rapporto alle proposte di Beltrami, ivi, 50-51; si deduce da questa analisi che la lettera, non datata, è comunque da trattenersi entro l'ottobre 1886. La relazione cui allude Beltrami è datata 28 giugno 1886, cfr. Raccolta Beltrami, C III, 25.

9 La lettera, non datata, riconduce tuttavia al restauro del campanile di S. Gottardo in cui Beltrami è coinvolto, come commissario provinciale nel 1887 e cui dedica, in quello stesso anno, un breve scritto, cfr. Raccolta Beltrami, B III, 15; D III, 36.

10 Su biglietto intestato 'Architetto/Luca Beltrami/Milano/via Cernaia, 5'. 


\section{Commenta la sospensione della pubblicazione della Raccolta Vinciana. ${ }^{11}$}

doc. 6 - 1888, luglio:: ${ }^{12}$ Luca Beltrami a Carlo Ermes Visconti, cas. CC, fasc. 3

\section{Caro Visconti}

Sono stato a S. Satiro: interessanti assai quelle tracce di pittura e di architettura, e spero se ne faranno i rilievi esatti e minuti per poter decidere riguardo al restauro il quale si va un poco complicando, ${ }^{13}$ Intanto ti ringrazio di avermi messo al corrente: ora mi assento per 4 o 5 giorni da Milano, ma ritornando mi affretterò a visitare nuovamente i lavori. Intanto credimi tuo aff. Luca Beltrami.

doc. 7 - 1888, ottobre 21: Luca Beltrami a Carlo Ermes Visconti, ${ }^{14}$ cas. CC, fasc. 3

11 Si tratta della Raccolta Milanese di Storia, Geografia ed Arte che si pubblica a Milano, ove è fondata dallo stesso Beltrami, per soli due anni (1887-1888) sotto la direzione di Gentile Pagani, direttore dell'archivio storico civico.

12 L'indicazione del giorno è mancante nel documento.

13 Nel febbraio del 1888 il parroco della chiesa di S. Satiro, Carlo Locatelli, promuove presso i milanesi una sottoscrizione per il restauro della cappella ottagonale progettata da Bramante; ad integrare i membri della Fabbriceria, cui è delegata la cura dell'edificio, viene istituita una commissione che include, oltre agli avvocati Gaetano Agnelli e Natale Zucchi, lo stesso Visconti, cfr. AVSV, cas. 115, fasc. 1888. Si apprende da qui che la commissione aveva il compito di vigilare sui restauri «del lato esterno della cappella della B.V. Addolorata» secondo il progetto dell'architetto ingegnere Enrico Strada, fabbriciere, e la vigilanza dell'architetto Augusto Guidini. Beltrami viene interpellato; assolve un compito istituzionale come Delegato Regionale per i Monumenti della Lombardia alimentato da interessi di studio sulle figure di Bramante, Bramantino e Bergognone. Nel suo archivio sono state censite non solo la copia della circolare di sottoscrizione ma anche appunti autografi sull'edificio, sulle pitture ivi presenti e annotazioni, anche di molto successive, delle consultazioni d'archivio sul tema, Raccolta Beltrami, B III, 33; B IV, 1; C III, 27.

${ }^{14} \mathrm{Su}$ carta intestata 'Ministero dell'Istruzione. Direzione Generale delle Antichità e Belle Arti'. Beltrami in quel momento è Delegato Regionale per i Monumenti della Lombardia, cfr. Grifoni 1992, 282. 


\section{Caro Visconti}

Leggo nei giornali di costì la relazione del banchetto dato in onore del Brentano e il riassunto del discorso da te pronunciato in tale circostanza. Delle parole gentili a mio riguardo ti ringrazio di cuore, perché mi sono di conforto nel faticoso compito che mi sono imposto di digerire nel silenzio e con cristiana rassegnazione il verdetto del Giurì. ${ }^{15}$ Credimi sempre tuo aff. Luca Beltrami.

doc. 8 - 1889, novembre 9: Luca Beltrami a Carlo Ermes Visconti ${ }^{16}$ cas. CC, fasc. 3

Chiede notizie sul trasporto della porta di Corso Magenta ${ }^{17}$ e avvisa di 'ritrovamenti' alle Grazie.

doc. 9 - s.d. ma ante 1890, febbraio 2, ${ }^{18}$ Luca Beltrami a Carlo Ermes Visconti, cas. CC, fasc. Autografi

Chiede che, come lui, anche l'amministrazione della Fabbrica del Duomo respinga la richiesta di illuminare il Duomo nel periodo di Carnevale. $^{19}$

doc. 10 - 1890, febbraio 2: Luca Beltrami a Carlo Ermes Visconti, cas. CC, fasc. Autografi

15 Si tratta ovviamente del Giurì del concorso di $2^{\circ}$ grado per il completamento della facciata del Duomo di Milano il cui verdetto è reso pubblico il 27 ottobre 1888. La lettera ufficiale con cui gli Amministratori della Veneranda Fabbrica, Carlo Ermes Visconti, Cesare Ajroldi ed Emilio Borromeo, informeranno Beltrami dell'esito è del 14 novembre 1888, cfr. Raccolta Beltrami, A IV, 33. La lettera trascritta conferma l'immediata reazione di Beltrami; il banchetto dato in onore di Brentano e il discorso di Visconti risalgono infatti al precedente giovedì, 18 ottobre, cfr. La Perseveranza, 20 ottobre 1888. Visconti a sua volta risponderà a Beltrami il successivo $1^{\circ}$ novembre, cfr. Raccolta Beltrami, A IV, 33.

16 Su carta listata a lutto.

17 Tra il 1881 e il 1904 l'area intorno alla chiesa delle Grazie è interessata da un intervento di isolamento che include l'apertura di via Caradosso ad est dell'edificio, cfr. Bortolotto, Massari 1992. Nel corso dei lavori si rinvenne un canale di fognatura realizzato con marmi e lapidi antiche. Beltrami studiò con Vincenzo Forcella i pezzi pubblicando, in particolare, proprio i rilievi del presunto lavabo citato nella lettera; Beltrami, Forcella 1989, 959-965.

18 Si data in rapporto al successivo doc. 23.

19 cfr. AFD, VII, 548 per la risposta, negativa, che daranno anche gli Amministratori della Veneranda Fabbrica. 
Sull'illuminazione del Duomo nel periodo di Carnevale conferma il diniego prefettizio.

doc. 11 - s.d. ma ante 1890, novembre 18:20 Luca Beltrami a Carlo Ermes Visconti, ${ }^{21}$ cas. CC, fasc. 3

Raccomanda il figlio di Bardeaux ${ }^{22}$ candidato ad un posto presso la Cassa di Risparmio e chiede di intercedere presso Annoni. ${ }^{23}$

doc. 12 - 1892, febbraio 9: Luca Beltrami a Carlo Ermes Visconti, cas. CC, fasc. 2

Allude alla 'resurrezione' di un una certa Commissione.

doc. 13 - 1892, aprile 8: Giovanni Celoria a Carlo Ermes Visconti, ${ }^{24}$ cas. CC, fasc. 1

Ringrazia per le informazioni ricevute sul Museo di Storia Naturale.

doc. 14 - 1892, giugno 30: Milano, Giunta Municipale del Comune di Milano a Carlo Ermes Visconti - Lettera circolare n. 44006515 Rip. VI, cas. CC, fasc. 5

${ }^{20}$ La datazione è dedotta dall'intestazione della carta. Vedi nota seguente.

$21 \mathrm{Al}$ verso del cartoncino è presente la dicitura 'Arch. Luca Beltrami/Professore presso il R. Istituto Tecnico Superiore'. Si ricorda che Beltrami subentra ad Archimede Sacchi (deceduto il 21 luglio 1886) nel ruolo di professore Straordinario di architettura pratica con decreto regio 4 novembre 1887; già nel corso del 1886 però lo suppliva. Rassegnerà le dimissioni dal Politecnico il 18 novembre 1890; Ricci 1997, 149.

22 Verosimilmente Carlo Bardeaux, economo della Consulta del Museo Patrio di Archeologia.

23 Il conte Aldo Annoni (1831-1900), Senatore del Regno, era in quel momento presidente del Consiglio di Amministrazione della Cassa di Risparmio oltre che consigliere municipale, cfr. Atti parlamentari 1900.

24 Giovanni Celoria (1842-1920) è da poco assessore del riparto VI subentrato a Visconti nel 1889, cfr. Janiro 1979. 


\section{Illustrissimo Signore}

Nella imminenza della consegna al Comune, per parte dell'Autorità militare, dell'intero fabbricato costituente il Castello si rende manifesta la necessità nell'Amministrazione municipale di provvedere per tempo alle diverse destinazioni a darsi a quel vasto e istorico edificio, epperò la Giunta è venuta nell'avviso di costituire una nuova ed unica Commissione nella quale concentrando anche gli elementi delle diverse che prima d'ora erano state a tale scopo create, avesse per iscopo [sic] di studiare:

I. In quali modi utilizzare l'edificio del Castello col destinarne le varie sue parti ad accogliere:

a) L'Archivio storico municipale e la Società Storica Lombarda;

b) Il Museo patrio d'archeologia;

c) Il Museo artistico municipale con l'annessa Scuola superiore d'arte applicata all'industria;

d) Il Museo civico del Risorgimento;

e) Il Corpo di musica municipale e le annesse Scuole Popolari di musica.

II. Se il ristauro [sic] interno ed esterno delle diverse parti costituenti il Castello possa svolgersi ed attuarsi parallelamente alla utilizzazione graduale delle singole parti.

Nella sua seduta del 27 corrente la Giunta municipale deliberava la costituzione della Commissione colle persone seguenti:

Marchese Carlo Ermes Visconti, conservatore del Museo artistico municipale e della Scuola superiore d'arte e membro della Commissione del Museo del Risorgimento, quale Presidente;

Architetto Luca Beltrami, Deputato del Parlamento, Ispettore ${ }^{25}$ Regionale dei monumenti nazionali in Lombardia;

Nobile Carlo D'Adda, Presidente della Commissione del Museo del Risorgimento;

Colonnello Enrico Guastalla, membro della Commissione suddetta;

Conte Emilio Belgiojoso, membro delegato della Consulta del Museo archeologico;

Marchese Emilio Visconti Venosta, Presidente della Regia Accademia di belle arti;

Nobile Felice Calvi, Vice Presidente della Società storica lombarda e membro della Commissione del Risorgimento;

Avvocato Emilio Seletti, Segretario della Società storica lombarda;

Cavaliere Antonio Besana, Direttore del Consiglio d'amministrazione del Corpo di musica municipale;

Cav. Gaetano Frizzoni, storico dell'arte;

25 Erroneamente indicato poichè l'esatta qualifica è Direttore (Delegato) compito di cui è incaricato il 31 ottobre 1885 . Fuorviante, più avanti nel testo, anche l'indicazione del nome di Frizzoni, più correttamente da intendersi Gustavo. 
conte Aldo Annoni, Senatore del Regno, Consigliere comunale;

Comm. Tullo Massarani, Senatore del Regno, Consigliere Comunale;

Nobile Gerolamo Sala, Consigliere comunale;

Quale Segretario il Nob. Dr. Giuseppe Luini, Segretario municipale.

+ Rosmini avv. Comm. Enrico

Nel dare communicazione [sic] alla S.V. Illustrissima della di Lei nomina a Presidente della Commissione in discorso, la Giunta nutre viva lusinga che V.S. sarà per accettare cortesemente tale ufficio ed in tale fiducia si riserva di farle conoscere quando avrà luogo e dove la prima convocazione della Commissione.

Aggradisca, Illustrissimo Signore, l'attestato del mio distinto ossequio. Pel Sindaco Fano.

Minuta di risposta in data 1 luglio 1892

Illustrissimo Signor Sindaco

Ho accolto con gioia l'annuncio datomi dalla S.V.I. colla nota 30 giugno n. 44006-515 Rip. VI. che l'On. Giunta Municipale mi ha chiamato a fare parte di una Commissione per la destinazione dei locali del Castello a scopo scientifico ed artistico, vedendo in tale atto della rappresentanza comunale un primo e serio passo alla realizzazione di un progetto che accarezzo da parecchi anni e che ha subito nel mio pensiero tutte le fasi delle cose fortemente amate e desiderate. E però accetto non solo senza reticenza ma con vera gratitudine tale nomina. Altrettanto non posso dire per la designazione della Presidenza di detta Commissione nella mia persona, prima perché tutti gli egregi membri che mi furono dati compagni mi soverchiano per posizione e per competenza poi - Lo confesso perché nella parte assegnatami di regolatore delle opinioni altrui vedo preclusa la via ad esprimere le mie nell'argomento. Colla maggiore considerazione. Devot.o C. E. Visconti. ${ }^{26}$

doc. 15 - 1892, novembre 8: Giovanni Celoria a Carlo Ermes Visconti, ${ }^{27}$ cas. CC, fasc. 1

Accordi per convocare la commissione per il Castello.

26 Le cancellature sono nel testo.

27 Su carta intestata 'Municipio di Milano'. 
doc. 16 - 1892, novembre 9: Milano, Il Sindaco del Municipio, Giuseppe Vigoni a Carlo Ermes Visconti. Lettera n. 44006/515 Rip. $\mathrm{VI},{ }^{28}$ cas. CC, fasc. 5

Convocazione della commissione per il Castello.

doc. 17 - 1892, novembre 17: Milano, Il Sindaco del Municipio, Giuseppe Vigoni a Carlo Ermes Visconti. Lettera n. 44006/515 Rip. $\mathrm{VI},{ }^{29}$ cas. CC, fasc. 5

Invia lascia passare per accedere al Castello.

doc. 18 - 1892, novembre 22: Milano, D. Luini a Carlo Ermes Visconti, cas. CC, fasc. 5

Illustrissimo Presidente

Ho mandato stamane a Brera per ritirare i disegni del Castello, ma venne risposto da quelli dello studio Beltrami ${ }^{30}$ che non avevano ordine, che però indicando loro quali disegni occorrevano al Municipio avrebbero procurato di farli avere. Cosa faccio? Non sarebbe miglior partito scrivere direttamente al Deputato a Roma? ${ }^{31}$

Attendo i tuoi pregiati ordini e mi dico Devotissimo D.G. Luini.

[E' allegata la seguente nota in altra grafia]

Locali occorrenti per la sede del Corpo di musica e per le Scuole popolari di musica.

n. 1 locale per la direzione da m. $4 \times 4$ circa

n. 1 locale per la Cancelleria e Copisteria da m. $4 \times 5$ circa

n. 1 locale per l'archivio e Biblioteca da m. 7x8 circa

n. 1 locale grandissimo per le ripetizioni del Corpo di musica da m. 12x12 circa

n. 5 locali per le scuole d'istrumenti da $\mathrm{m} .3 \times 4$ cad. circa

n. 3 locali per le scuole di canto corali da m. 10x12 circa cadauno

ก. 12

28 Su carta intestata 'Municipio di Milano'.

29 Su carta intestata 'Municipio di Milano'.

30 Si tratta dell'Ufficio Regionale che Beltrami dirige dal 1 ottobre 1891 e il cui nome evidentemente basta ad identificare l'ufficio, cfr. Bellini 1997b, 100.

31 Il Deputato in questione è Luca Beltrami, cfr. Bellini 1997b, 110. 
N.B. Nel locale della Direzione si potrebbe collocare la Biblioteca ed in quello della Cancelleria e Copisteria l'Archivio, purché siano più grandi delle misure segnate sopra. Il locale per la ripetizione del Corpo di musica potrebbe servire per un corso sia maschile che femminile della Scuola di canto corale. Percui anziché 12 locali potrebbero essere sufficienti 9 locali, senza tener conto dei locali occorrenti per il portiere - custode e per il magazzeno che in tutto ce ne vorrebbero altri 4 da circa m. $4 \times 4$ cadauno. Allievi ed allieve delle Scuole n. 375. Musicanti e coristi 60. [Totale] 435.

doc. 19 (289) -1892, dicembre 2: Milano, Gaetano Moretti a Carlo Ermes Visconti, ${ }^{32}$ cas. CC, fasc. 5

Accusa ricevuta delle tavole planimetriche del Castello.

doc. 20 (270) - 1892, dicembre 16: Luca Beltrami a Carlo Ermes Visconti, cas. CC, fasc. 1

Ricorda un quadro raffigurante il Castello in possesso del commendatore Fuzier, ${ }^{33}$ ora deceduto, e già riprodotto da Carlo Casati ${ }^{34} \mathrm{e}$ chiede di interessarsi per l'acquisto da parte del Municipio.

doc. 21 - 1892, dicembre 24: Luca Beltrami a Carlo Ermes Visconti, cas. CC, fasc. 5

\section{Caro Visconti}

Sono a Milano da jeri e sino al 10 gennaio non ho alcun impegno d'importanza per cui tu potrai disporre liberamente per la convocazione della Commissione del Castello. Auguri sinceri a te e a tutta l'egregia tua famiglia dall'aff. L. Beltrami.

[a margine:] Ho visto che l'eredità Fuzier è in mano dell'avv. Cologna e avv. Garavaglia per cui sarà possibile assicurare il quadro del Castello. ${ }^{35}$

32 per esteso: Gaetano Moretti p. il Direttore dell'Ufficio Regionale per la conservazione dei Monumenti di Lombardia all'Ill.mo Signor Visconti marchese Carlo Ermes, Presidente della Commissione Municipale per il Castello di Milano.

33 Si tratta del commendatore Luigi Fuzier deceduto il 13 dicembre 1892, La Perseveranza, 15 dicembre 1892.

34 Si allude a Casati 1876. Per i contenuti del volume, anche in rapporto alla storiografia sul castello, cfr. Di Biase 1997, 37.

35 Con testamento 15 dicembre 1888 in atti del notaio Stefano Allocchio di Milano, Luigi Fuzier aveva espresso la volontà «che tutti gli oggetti di arte antica e 
doc. 22 - 1892, dicembre 28: Il Presidente Carlo Ermes Visconti a Carlo Ermes Visconti da Milano - Lettera circolare n. 91536-953 Rip. $\mathrm{VI},{ }^{36}$ cas. CC, fasc. 5

La Commissione istituita per gli studi di collocamento nel Castello di varie istituzioni cittadine viene convocata in adunanza pel giorno di Martedì 3 Gennaio p.v. alle ore 3 pom. al civico palazzo Marino nella Sala della Soprintendenza Scolastica.

La S.V. Ill.ma qual'altra dei componenti la Commissione suddetta è pregata del suo intervento a detta adunanza. Il Presidente C.E.Visconti.

doc. 23 - 1893, gennaio 5: Luca Beltrami a Carlo Ermes Visconti da Milano, ${ }^{37}$ cas. CC, fasc. 5

Caro Visconti

Siccome probabilmente dovrò fare una corsa a Roma presto, così sarebbe bene che prima di partire ci trovassimo coll'arch.o Arcaini ${ }^{38}$ per dare a questi le istruzioni. Se quindi domani, venerdì o sabato verso le 10 ant. ti fa comodo di passare all'Ufficio qui a Brera ci troverai: oppure fissaci un'altra ora di tuo comodo. Aff.o Beltrami.

doc. 24 - 1893, gennaio 11: D.G. Luini a Carlo Ermes Visconti da Milano, cas. CC, fasc. 5

Informa che l'autorità militare intende concedere spazi in Castello ad uso della commissione incaricata di valutare la prossima destinazione d'uso dell'edificio.

moderna da lui posseduti in Milano fossero venduti, per asta pubblica, entro un anno dalla sua morte, e il ricavo netto destinato in parti eguali a quattro Istituti da lui designati». Tra i beneficiari del lascito vi fu la Società d'Incoraggiamento d'arti e mestieri di Milano che fu autorizzata ad accettare la somma ricavata il 19 marzo 1893, in Gazzetta 1893, 1674.

36 Su carta intestata 'Municipio di Milano'.

37 Su carta intestata 'Ufficio Regionale per la conservazione dei monumenti della Lombardia'.

38 Si tratta di Raineri Arcaini 'rappresentante' dell'Ufficio Regionale, la cui presenza nell'ufficio è registrata, come disegnatore, solo a partire dal 1896, cfr. Grifoni 1992, 293. Il documento dunque chiarisce già da questa data oltre a questo ruolo, anche quello, se pure ufficioso, di vigilanza dei lavori. 
doc. 25 - 1893, gennaio 18: D. Luini a Carlo Ermes Visconti da Milano, ${ }^{39}$ cas. CC, fasc. 5

Trasmette convocazione del Sindaco per discutere di una parziale occupazione di locali in Castello.

doc. 26 - 1893, gennaio 21: D. Luini a Carlo Ermes Visconti da Milano, ${ }^{40}$ cas. CC, fasc. 5

Informa degli avvenuti accordi con l'autorità militare per occupazione locali del castello ad uso della Commissione.

doc. 27 - 1893, gennaio 25: D.S. Luini a Carlo Ermes Visconti da Milano, cas. CC, fasc. 5

Avvisa che ha preso possesso, ad uso della commissione, del locale n. 54 in Castello.

doc. 28 - 1893, gennaio 31: Rainieri Arcaini a Carlo Ermes Visconti da Milano, ${ }^{41}$ cas. CC, fasc. 5

\section{Egregio Signore}

E' necessario avvertire d'urgenza e d'ufficio l'Imp.a Bonomi ${ }^{42}$ di risparmiare nelle demolizioni del Muraglione N-O la parte antica ora scoperta, e che Ella ben conosce. Per conto nostro ordinaj all'Ass.e dell'Impresa di puntellare tosto le mensole spezzate per evitare che cadano per gli effetti delle prossime mine. Il rappresentante dell'Ufficio Regionale a cui fu fin d'ora affidata l'operazione dei rilievi e la protezione delle opere antiche che si rinvengano nelle demolizioni, dovrebbe avere facoltà di ordinare l'immediata sospensione delle demolizioni delle parti che si devono conservare; e questo per evitare qualunque indugio che potrebbe compromettere l'esistenza di una preziosa costruzione; se si deve tener conto anche che queste demolizioni vengono eseguite a cottimo da operai che torna lor conto di celare qualunque indizio che possa loro incagliare il procedere del lavoro. Con distinta stima. Dev. R. Arcaini arch.to.

39 Per esteso: D.r. Luini, segretario, all'illustrissimo sig. Marchese Carlo Ermes Visconti. Su carta intestata 'Comune di Milano. Soprintendenza Scolastica'.

40 Su carta intestata 'Municipio di Milano'.

${ }^{41} \mathrm{Su}$ carta intestata 'Ufficio Regionale per la conservazione dei monumenti della Lombardia'.

42 Si tratta della ditta Bonomi appaltatrice delle opere di demolizione. In merito ai contenziosi insorti con la ditta, alle rimostranze di Arcaini e ai commenti di Beltrami stesso nella successiva costruzione della torre Umberto I, cfr. Biase 1997, $51-52$ e Raccolta Beltrami, A IV, 5. 
doc. 29 - 1893, marzo 27: Luini, segretario della $[\ldots]^{43}$ a Carlo Ermes Visconti Milano, ${ }^{44}$ cas. CC, fasc. 5

Lo convoca ad una prossima seduta della Commissione per l'assegnazione dei locali in Castello.

doc. 30 - 1894, febbraio 19: Luca Beltrami a Carlo Ermes Visconti, cas. CC, fasc. 3

Caro Visconti

Desidererei che tu avessi ad esaminare da vicino i lavori della merlatura del torrione: la mia voce vale poco presso il Sindaco: forse tu potresti ottenere che si provveda a fare un lavoro un poco più decente. Ti saluto aff. Beltrami

doc. 31 - 1894, agosto 4: Giovanni Celoria a Carlo Ermes Visconti, cas. CC, cronologia castello

Rende, ringraziando, i manoscritti riguardanti pratiche e studi sul Castello.

doc. 32 - 1894, ottobre 16: Luca Beltrami ${ }^{45}$ a Carlo Ermes Visconti, cas. CC, fasc. 3

In merito ad una richiesta di locali avanzata dalla Regia Accademia di Brera esprime anch'egli parere favorevole.

doc. 33 - 1894, ottobre 26: Il Com. straordinario del [...] a Carlo Ermes Visconti Milano, cas. 116.

Approssimandosi la chiusura delle Esposizioni Riunite chiede di convocare la Commissione per il Castello.

doc. 34 - 1894, novembre 28: Luca Beltrami a Carlo Ermes Visconti, ${ }^{46}$ cas. CC, fasc. 3 (Fig. 1)

43 Mancante nel documento.

44 Su carta intestata 'Municipio di Milano. Ufficio Commissione Castello'.

45 Su carta intestata 'Ufficio Regionale per la conservazione dei monumenti della Lombardia'.

$46 \mathrm{Su}$ carta intestata 'Ufficio Regionale per la conservazione dei monumenti della Lombardia'. 


\section{Caro Visconti}

Fui oggi in Castello dove vidi il Müller ${ }^{47}$ nella Sala della Torre che ha trovato il principio della decorazione della volta, costituito da grossi tronchi d'albero. Egli mi accennò che per conservare la decorazione occorre molto fissativo, ${ }^{48}$ lasciandomi capire che la spesa sarebbe di qualche centinajo di lire per tutta la sala: mi accennò pure alle spese già incontrate da lui pel Castello. Non ti pare che si potrebbe vedere il modo di scaricare il Müller da quelle spese materiali che egli deve incontrare a vantaggio del monumento. Io potrei fare qualcosa col fondo dell'Ufficio, se anche il Comune vi concorresse. Ti dico ciò perché avendo occasione di andare in Castello potresti tu pure discorrerne col Müller e poi vedere in che modo provvedere. Cordiali saluti aff. L. Beltrami.

doc. 35 - 1894, dicembre 26: Luca Beltrami a Carlo Ermes Visconti, ${ }^{49}$ cas. CC, fasc. 3 (Fig. 2)

\section{Caro Visconti}

Il D.r Müller ha trovato delle tracce importanti di pitture nella Sala del Tesoro (Rocchetta) anteriori a Lod.o il Moro - maniera di V. Foppa. Cordiali saluti e auguri aff. L. Beltrami.

doc. 36 - s.d.: Luca Beltrami a Carlo Ermes Visconti, cas. CC, fasc. 2 Accordi per sopralluogo in Castello con Gaetano Moretti.

47 Paul Müller-Walde (1858-1931) per la cui figura si veda Costa 2006. Per il restauro della Sala della Asse cfr. ora Catturini 2013 con bibliografia precedente tra cui, in particolare, Fiorio, Lucchini, 2007.

48 Sottolineato nel testo.

49 Su carta recante la riproduzione del disegno del fronte del Castello di Milano. 